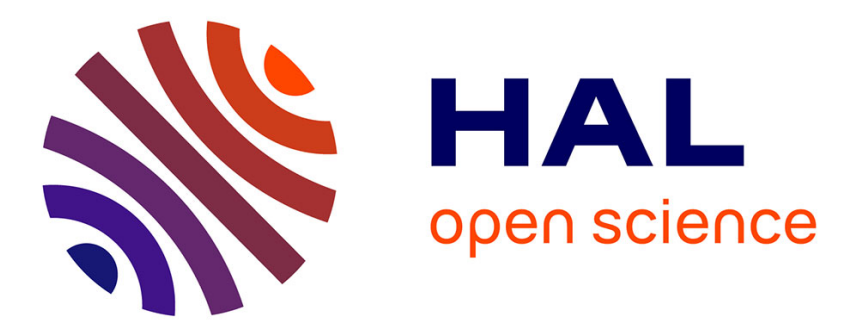

\title{
Molecular Dissection of the Interface between the Type VI Secretion TssM Cytoplasmic Domain and the TssG Baseplate Component
}

Laureen Logger, Marie-Stéphanie Aschtgen, Marie Guérin, E. Cascales, Eric Durand

\section{To cite this version:}

Laureen Logger, Marie-Stéphanie Aschtgen, Marie Guérin, E. Cascales, Eric Durand. Molecular Dissection of the Interface between the Type VI Secretion TssM Cytoplasmic Domain and the TssG Baseplate Component. Journal of Molecular Biology, 2016, 428 (22), pp.4424 - 4437. 10.1016/j.jmb.2016.08.032 . hal-01780174

\section{HAL Id: hal-01780174 \\ https://hal-amu.archives-ouvertes.fr/hal-01780174}

Submitted on 27 Apr 2018

HAL is a multi-disciplinary open access archive for the deposit and dissemination of scientific research documents, whether they are published or not. The documents may come from teaching and research institutions in France or abroad, or from public or private research centers.
L'archive ouverte pluridisciplinaire HAL, est destinée au dépôt et à la diffusion de documents scientifiques de niveau recherche, publiés ou non, émanant des établissements d'enseignement et de recherche français ou étrangers, des laboratoires publics ou privés. 


\title{
Molecular dissection of the interface between the Type VI secretion TssM cytoplasmic domain and the TssG baseplate component.
}

\author{
Laureen Logger ${ }^{1}$, Marie-Stéphanie Aschtgen ${ }^{\Uparrow}$, Marie Guérin ${ }^{\dagger}$, Eric Cascales ${ }^{1, *}$, and Eric \\ Durand $^{1, *}$
}

Laboratoire d'Ingénierie des Systèmes Macromoléculaires, Institut de Microbiologie de la Méditerranée, Aix-Marseille Université, CNRS - UMR 7255, 31 chemin Joseph Aiguier, 13402 Marseille Cedex 20, France.

Running head: T6SS baseplate-TssM interactions

Present addresses:

` Laboratoire des Sciences de l'Environnement Marin (LEMAR), Institut Universitaire Européen de la Mer (IUEM), Université de Bretagne Occidentale, CNRS, IRD, Ifremer - UMR 6539, Technopôle Brest Iroise, 29280 Plouzané, France.

${ }^{\dagger}$ Maquet Intervascular, 13600 La Ciotat, France

* Address correspondence to Eric Durand, edurand@imm.cnrs.fr or Eric Cascales, cascales@imm.cnrs.fr 


\begin{abstract}
The type VI secretion system (T6SS) is a multi-protein complex that catalyses toxin secretion through the bacterial cell envelope of various Gram-negative bacteria including important human pathogens. This machine uses a bacteriophage-like contractile tail to puncture the prey cell and inject armful toxins. The T6SS tail comprises an inner tube capped by the cell-puncturing spike and wrapped by the contractile sheath. This structure is built on an assembly platform, the baseplate, which is anchored to the bacterial cell envelope by the TssJLM membrane complex. This membrane complex serves both as tail docking station and channel for the passage of the inner tube. The TssM trans-membrane protein is a key component of the membrane complex as it connects the inner and outer membranes. In this study, we define the TssM topology, highlighting a large but poorly studied $35 \mathrm{kDa}$ cytoplasmic domain, TssM $\mathrm{M}_{\mathrm{Cyt}}$, located between two trans-membrane segments. Proteinprotein interaction assays further show that $\mathrm{TssM}_{\text {Cyto }}$ oligomerizes and makes contacts with several baseplate components. Using computer predictions we delineate two sub-domains in TssM $_{\text {Cyto }}$, including a nucleotide tri-phosphatase (NTPase) domain followed by a 110-aminoacid extension. Finally, site-directed mutagenesis coupled to functional assays reveal the contribution of these sub-domains and of conserved motifs to the interaction with T6SS partners and to the function of the secretion apparatus.
\end{abstract}

Keywords: protein transport, protein secretion, Type VI secretion, bacterial competition, membrane complex. 


\section{INTRODUCTION}

The Type VI secretion system (T6SS) is a versatile multi-protein secretory machine that is implicated in both inter-bacterial competition and anti-eukaryotic host activities. The T6SS delivers a broad arsenal of toxins with peptidoglycan, phospholipid or DNA hydrolysis activities, or that induce cytoskeleton re-arrangements directly into the target cell. ${ }^{1-4}$

For toxin delivery, the T6SS uses a contractile mechanism that is comparable to that of Myoviridae phages or R-pyocins. ${ }^{5-10}$ This machine is composed of 13 core subunits, categorized in three sub-complexes ${ }^{8,10-12}$ : a cytoplasmic tubular structure built on an assembly platform - or baseplate (BP) - that is evolutionarily, structurally and functionally related to bacteriophage contractile tails ${ }^{5,13-15}$, and anchored to the cell envelope by a membrane complex (MC). ${ }^{16}$

The T6SS tail is composed of an inner tube made of stacked Hcp hexameric rings and wrapped into a sheath-like structure, formed by the polymerization of TssB-TssC heterodimeric complexes, and that is assembled in an extended conformation. ${ }^{14,17-19}$ Indeed, the assembly of the tail can be followed by time-lapse microscopy: fluorescent-labelled sheath components assemble $\mathrm{a} \sim 600 \mathrm{~nm}$-long tubular structure in tens of seconds, that then contracts in a few millisec. ${ }^{14,20}$ The contraction of the sheath coincides with bacterial prey lysis, suggesting that, similarly to phages, sheath contraction propels the inner tube towards the target cell, allowing delivery of toxin effectors). ${ }^{8,11,20,21}$ The assembly of the tube and the sheath is coordinated by TssA, a protein that controls the elongation of the tail at the distal end and that maintains the sheath under the extended conformation. ${ }^{22}$ The inner tube is tipped by a spike constituted of a trimer of the VgrG protein, which is proposed to puncture the target cell membrane. ${ }^{13,23}$ The VgrG trimer is also part of the BP that is used as an assembly plateform for the tail. Recently, the T6SS BP composition has been revealed. In addition to VgrG, it is composed of the TssE, -F and -G subunits, the homologues of the phage T4 gp25, gp6 and gp7 proteins, respectively, as well as of TssK a protein of unknown function with limited homologies to phage $\mathrm{T} 4 \mathrm{gp} 8$ or gp10 proteins that has been proposed to be a connector to the membrane complex. ${ }^{15,24-27}$ This MC is composed of the two TssL and TssM inner membrane (IM) proteins and of the TssJ outer membrane (OM) lipoprotein. ${ }^{28-32}$ TssL and TssM interact in the IM whereas the C-terminal periplasmic domain of TssM contacts the Tss J lipoprotein close to the OM. ${ }^{16,29,30,33-35}$ The MC serves as a docking station for the BP 
and the tail, but has also been proposed to serve as channel for the passage of the inner tube during sheath contraction. ${ }^{16}$ In the recent years, the assembly pathway of the T6SS has been well defined. T6SS biogenesis progresses from the outer membrane to the cytoplasm. It starts with the positioning of the TssJ lipoprotein and the successive recruitments of TssM and TssL. ${ }^{16}$ Recruitment of TssA then positions the baseplate complex onto the MC and primes the polymerization of the tail tube/sheath. ${ }^{15,22,36}$ This ordered assembly pathway requires tight contacts between the different subunits. Indeed, docking of the BP onto the MC requires multiple contacts including interactions of TssE and TssK with TssL, and of TssG and TssK with TssM. ${ }^{15,24}$ TssM is therefore a key component as it mediates contact with the OM TssJ lipoprotein, as well as with cytoplasmic BC subunits.

Here, we show that the enteroaggregative Escherichia coli TssM protein is a polytopic membrane protein, inserted into the inner membrane by three trans-membrane helices (TMH). The C-terminal portion of TssM is in the periplasm and interacts with TssJ. ${ }^{34}$ TMH2 and TMH3 delimitates a $\sim 35 \mathrm{kDa}$ cytoplasmic domain, TssM $_{\text {Cyto }}$, which is conserved among TssM homologues. Computer analyses show that $\mathrm{TssM}_{\text {Cyto }}$ is constituted of two sub-domains: a sub-domain with a nucleotide tri-phosphatase (NTPase)-like domain followed by an extension. Indeed, TssM has been previously shown to bind and hydrolyse NTPs. ${ }^{37}$ However, the role of the NTP-binding motif and its functional implication during T6SS activity is still a matter of debate. ${ }^{29,33}$ The extension comprises a eukaryotic DPY-30-like dimerization motif. We show that the NTPase-like domain mediates interaction with TssK whereas the extension is necessary and sufficient for $\mathrm{TssM}_{\mathrm{Cyto}}$ oligomerization and interaction with TssG. Sitedirected mutagenesis of conserved motifs within the extension revealed their contribution for TssM $_{\text {Cyto }}$ oligomerization, TssM $\mathrm{Cyto}_{\text {Cyto }}$ TssG interaction and for proper assembly of the T6SS. Our results thus provide details on the molecular interface between the T6SS membrane and baseplate complexes.

\section{RESULTS}

\section{TssM is a polytopic IM protein}

The TssM protein encoded within the enteroaggregative E. coli sci-1 gene cluster (EC042_4539; Genbank accession (GI): 284924260) is a large protein of 1129 amino acids. 
Based on hydrophobicity plots, most widely used computer tools predict TssM as an inner membrane protein with three trans-membrane helices (TMH) (Fig. 1A). Indeed, fractionation experiments showed that TssM co-fractionates with membrane proteins (data not shown). To experimentally define the TssM topology and determine the TMH boundaries we performed a cysteine accessibility assay using the substituted cysteine accessibility method (SCAM, Ref 38. This assay relies on the ability of 3-( $N$-maleimidylpropionyl) biocytin (MPB), a sulfhydryl reagent to cross the outer membrane but not the inner membrane of Gram-negative bacteria including EAEC. ${ }^{30,31}$ TssM possesses nine native cysteine residues, with one $(\mathrm{C} 727)$ predicted to locate in the periplasm. Hence, the WT TssM protein is labelled by MPB in vivo (Fig. 1B). In agreement with the computer predictions, a TssM protein in which the cysteine at position 727 is substituted to serine (C727S) was not labelled with MPB (Fig. 1B). These data suggest that $\mathrm{C} 727$ is located in the periplasm whereas all other 8 cysteine residues locate in the cytoplasm or are buried into the structure of the protein and then inaccessible to MPB. We then introduced cysteine substitutions in the C727S TssM variant at various positions along the protein (at positions 37, 67, 352 and 386) (Fig. 1A). All these mutated proteins were produced at similar levels (Fig. 1B) and were able to complement the effect of the tss mutant in an Hcp secretion assay (data not shown). The A37C and S386C variants were biotinylated with MPB suggesting that the A37 and S386 residues locate in the periplasm (Fig. 1B). By contrast, the V67C and S352C variants were not labelled indicating that the V67 and S352 residues locate in the cytoplasm (Fig. 1B). All together, the data of the cysteine accessibility defined the topology of TssM: TssM is constituted of three TMH, with the Nterminus in the cytoplasm and the C-terminus in the periplasm. TssM spans the IM through two TMH oriented in-to-out (TMH1, residues 13-29; TMH3, residues 360-382) and one TMH oriented out-to-in (TMH2, residues 44-62) (Fig. 1C). TMH2 and TMH3 thus delimitate a $~ 35$ $\mathrm{kDa}$ domain located in the cytoplasm, called hereafter $\mathrm{TssM}_{\mathrm{Cyto}}$.

\section{The cytoplasmic domain of TssM oligomerizes and interacts with components of the T6SS membrane and baseplate complexes}

The topology of TssM indicates the existence of two soluble domains, one in the periplasm (TssM $M_{\text {Peri }}$, amino-acids 383-1129) and one residing into the cytoplasm (TssM $\mathrm{Cyto}_{\text {, }}$ amino-acids 63-359). The T6SS being a multi-protein complex, such large protein domains might be necessary for interacting with other T6SS components. Indeed, we and other have previously demonstrated that $\mathrm{TssM}_{\text {Peri }}$ interacts with the TssJ outer membrane lipoprotein in 
Edwardsiella tarda and enteroaggregative E. coli. ${ }^{33,34}$ By contrast, little is known regarding the cytoplasmic domain of TssM. To gain further insights onto TssM $\mathrm{Cyto}_{\text {to }}$ partners, we used $\mathrm{TssM}_{\text {Cyto }}$ as bait for an in vivo systematic bacterial two-hybrid assay. TssM $\mathrm{S}_{\text {Cyto }}$ was fused to the T18 domain of the Bordetella adenylate cyclase and all the other T6SS proteins - or soluble domains - were fused either at their N- or C-terminus of the T25 domain. The results presented in Fig. 2 show that TssM $_{\text {Cyto }}$ interacts with itself and with TssK whatever the constructions used. In addition, TssM $\mathrm{M}_{\text {Cyto }}$ interacts with TssG and with the cytoplasmic domain of TssL ( $\mathrm{TssL}_{\mathrm{Cyto}}$ ) when fused at the N-terminus of T25. In conclusion, $\mathrm{TssM}_{\text {Cyto }}$ is capable of oligomerization and interacts with components of the T6SS membrane (TssL $\left.L_{\text {Cyto }}\right)$ and baseplate (TssK and TssG) complexes. These results are in agreement with previously published bacterial two-hybrid screens and co-immune precipitations that identified TssMTssK, TssM-TssL and TssM-TssG interactions. ${ }^{15,24,29,30}$

\section{Sub-domain architecture of TssM $M_{\text {Cyto }}$}

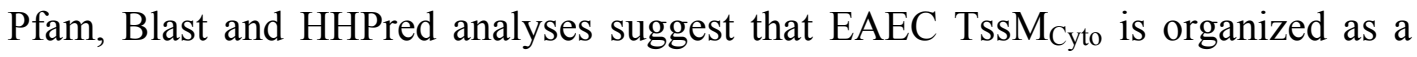

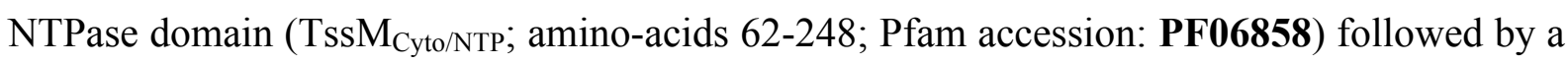
C-terminal extension (TssM ${ }_{\text {Cyto/Ct; }}$ amino-acids 254-360) (Fig. 3; Supp. Fig. S1-S2). However, despite the fact that the overall NTPase domain is conserved among the TssM $\mathrm{M}_{\text {Cyto }}$ homologs, the sequence alignment shows that the NTP binding and hydrolysis motifs (Walkers A \& B and NTP specific motif) are not well conserved (Supp. Fig. S1). In agreement with this observation, an evolutionary analysis of $\mathrm{TssM}_{\mathrm{Cyto}}$ NTPase domains shows that they categorize into two sub-groups: while the TssM proteins encoded within the Pseudomonas aeruginosa H1, Agrobacterium tumefaciens and Edwardsiella tarda T6SS gene clusters carry a complete NTPase domain, a number of TssM, including that of EAEC, Serratia and Citrobacter possess a NTPase domain amputated of hydrolysis motifs (Supp. Fig. S1 and S2).

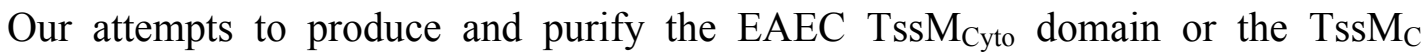
NTPase sub-domain in order to gain structural information were unsuccessful, as the different constructs used were all insoluble. Consequently, we sought to construct homology-based models of both $\mathrm{TssM}_{\text {Cyto }}$ sub-domains using a bioinformatic approach. The TssM $\mathrm{C}_{\text {Cyto/NTP }}$ structure was predicted using HHpred. ${ }^{39}$ The program confirmed that the EAEC TssM Cyto/NTP $_{\text {. }}$ protein resembles the solved structure of various GTP-hydrolysing proteins (Supp. Fig. S3). The X-ray structure of the Burkholderia thailandensis EngB GTP-binding protein (PDB ID: 4DHE $)^{40}$ was subsequently used as template to build a homology model of the EAEC 
TssM $_{\text {Cyto/NTP }}$ domain. Figure 3 shows that TssM $_{\mathrm{C} / \mathrm{NTP}}$ adopts a compact fold consisting of a four-stranded parallel $\beta$-sheet with one side being in contact with three $\alpha$-helices. The TssM $_{\text {Cyto/NTP }}$ domain belongs to the $\alpha / \beta$ class, harbouring an incomplete Rossmann fold, a motif associated with nucleotide-binding proteins. ${ }^{41}$ The EAEC TssM $\mathrm{Cyto}_{\mathrm{NTP}}$ architecture is typical of P-loop nucleotide triphosphate hydrolases. The predicted structure of EAEC

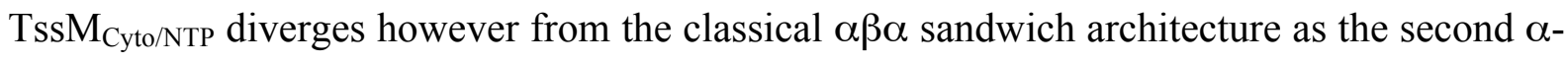
helix is not strictly sandwiching the $\beta$-strand. As expected, the large loops that bear the binding and hydrolysis motifs are absent in the EAEC TssM $\mathrm{Cyto}_{\mathrm{NTP}}$, by contrast to EngB or the homology model of the A. tumefaciens TssM $\mathrm{Cyto}_{\mathrm{NTP}}$ domain (Supp. Fig. S4). The TssM $_{\text {Cyto/Ct }}$ homology model was constructed using the Swiss-Model server based on the Xray structure of the C-terminal domain of human DPY-30-like protein, a component of the eukaryotic histone methyltransferase complex (PDB ID: 3G36) ${ }^{42}$ as template (Supp. Fig. S3). The TssM $\mathrm{C}_{\mathrm{C} / \mathrm{Ct}}$ structure was confidently modelled from residue Q254 to N289 (Fig. 3). This fragment encompasses the two-helix 40-amino-acid Dpy-30 motif (Pfam accession: PF05186) found in DPY-30 proteins and involved in DPY-30 dimerization ${ }^{42}$.

\section{Specific motifs are involved in TssM $M_{\text {Cyto }}$ oligomerization and interaction with TssG.}

The structural organization of $\mathrm{Tss}_{\mathrm{Cyto}}$ prompted us to investigate the contribution of the two sub-domains to the TssM $\mathrm{Cyto}_{\text {interactions. The interaction network of TssM }}$ Cyto/NTP and $\mathrm{TssM}_{\mathrm{Cyto} / \mathrm{Ct}}$ was assessed by bacterial two-hybrid. As shown in Figure 4A, TssM $\mathrm{Cyto}_{\mathrm{NTP}}$ interacts with $\mathrm{TssK}$, whereas $\mathrm{TssM}_{\mathrm{Cyto} / \mathrm{Ct}}$ mediates oligomerization and interactions with TssG and $\mathrm{TssL}_{\mathrm{Cyto}}$. Interestingly the interaction network of the isolated sub-domains, TssM $\mathrm{Cyto}_{\mathrm{NTP}}$ and $\mathrm{TssM}_{\mathrm{Cyto} / \mathrm{Ct}}$, recapitulates the interaction network of $\mathrm{TssM}_{\mathrm{Cyto}}$ (Fig. 4A), supporting the hypothesis of two independently-folded domains. The TssM $\mathrm{Cyto}_{\mathrm{Ct}}-\mathrm{Tss} \mathrm{G}$ and $\mathrm{TssM}_{\mathrm{Cyto} / \mathrm{NTP}}$ TssK interactions were further confirmed by co-immuno-precipitation experiments: TssG was co-precipitated with $\mathrm{TssM}_{\mathrm{Cyto}}$ and $\mathrm{TssM}_{\mathrm{Cyto} / \mathrm{Ct}}$ (Fig. 4B, upper panel). As previously shown ${ }^{24}$, TssK did not interact with TssM $_{\text {Cyto }}$ by co-immuno-precipitation. However, our results suggest that it is prevented by the extension as the TssM $\mathrm{Cyto}_{\mathrm{NTP}}$ domain alone interacts with TssK (Fig. 4B, lower panel).

Interestingly, the $\mathrm{Tss}_{\mathrm{Cyto} / \mathrm{Ct}}$ sub-domain interacts with several partners from the membrane and baseplate sub-complexes. We therefore questioned whether these different interactions involve the same recognition motif or different binding epitopes on $\mathrm{TssM}_{\mathrm{Cyto}}$. The 
sequence alignment of $\mathrm{TssM}_{\mathrm{Cyto}}$ homologues emphasized two well-conserved regions, F278E284 and L309-S315 (Supp. Fig. S1). Region F278-E294 is specifically conserved in

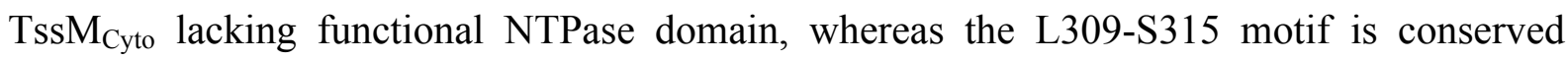
among all TssM $\mathrm{M}_{\text {Cyto }}$ (Supp. Fig. S1). Using site-directed mutagenesis, we engineered TssM $\mathrm{M}_{\text {Cyto }}$ variants in which these motifs were targeted. Although these two motifs do not appear to be involved in TssM $_{\text {Cyto }}-$ TssL $_{\text {Cyto }}$ interactions, substitutions within the L309-S315 motif specifically abolished the TssM $_{\text {Cyto }}-$ TssG interaction (Fig. 5A and 5B). We also noted that

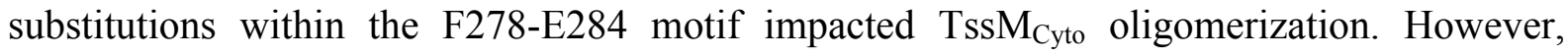
although the L279W and L282W/A283W mutations prevented interaction with TssM $\mathrm{M}_{\text {Cyto }}$ in the bacterial two-hybrid assay (Fig. 5A), only the L279W mutations had a strong effect on multimerization in the co-immunoprecipitation assay (Fig. 5B). It is worthy to note that the $\mathrm{TssM}_{\mathrm{Cyto} / \mathrm{Ct}} \mathrm{F} 278$-E284 residues correspond to the dimerization motif in Dumpy-30 (DPY-30) proteins (Supp. Fig. S4B).

\section{TssM $_{\text {Cyto }}$ oligomerization and interaction with the TssG baseplate subunit are critical for T6SS function.}

The mutations that specifically affect $\mathrm{Tss}_{\mathrm{Cyto}}$ oligomerization and $\mathrm{TssM}_{\mathrm{Cyto}}-\mathrm{Tss} \mathrm{G}$ complex formation were tested for their repercussion on T6SS function. EAEC Sci-1 T6SS

function could be monitored by measuring its antibacterial activity. ${ }^{43}$ The four TssM substitutions were introduced within the native, chromosomal tss $M$ gene. Fig. 6A shows that all four mutated strains were defective in T6SS-dependent killing of prey bacterial cells. We therefore conclude that $\mathrm{TssM}_{\mathrm{Cyto}}$ oligomerization and interaction with TssG are required for proper function of the Type VI secretion apparatus.

T6SS biogenesis starts with the assembly of the membrane complex and is followed by (i) the recruitment of the baseplate and (ii) tail polymerization. We therefore sough to define which stage of T6SS biogenesis is impacted by these mutations. We first tested the effects of these mutations on T6SS sheath assembly by following the dynamics of a chromosomally-encoded TssB-sfGFP fusion using fluorescence microscopy (Fig. 6B). All the substitutions severely affected T6SS sheath assembly as indicated by the decrease in the number of sheath per bacterial cell (Fig. 6B). While $\sim 25 \%$ of the wild-type cells assembled sheath structures, TssBC sheath assembled on rare occasions in cells carrying mutations affecting TssM $\mathrm{M}_{\text {Cyto }}$ oligomerization (3-4 \% of cells with sheath structures). The effect of the 
TssM $_{\text {Cyto }}$-TssG disruption was even more drastic as sheath assembly was observed in $\sim 1 \%$ of the cells.

Second, we tested the effect of these mutations on recruitment of the baseplate. By following the dynamics of a sfGFP-TssF fusion, a recent study concluded that the membrane complex recruits and stabilizes the T6SS baseplate. ${ }^{15}$ We therefore investigated whether the mutations affecting oligomerization of the cytoplasmic loop of TssM and contacts between this loop and the TssG baseplate component impact baseplate assembly, stability and recruitment. As TssG fusions to sfGFP were previously shown to be non functional ${ }^{15}$, we introduced the TssM point mutations in a strain producing the chromosomal and functional sfGFP-TssF fusion, a TssG protein partner, and we monitored the formation and stability of baseplate foci by fluorescence microscopy (Fig. 6C). All four mutations significantly decreased the number of cells with sfGFP-TssF foci and the average number of foci per cell ( 1 cluster in $15-25 \%$ of the mutated cells compared to $1-3$ foci in $\sim 65 \%$ for WT cells) (Fig. 6C and 6D). Taken together, these data demonstrate that mutations that affect TssM $\mathrm{M}_{\text {Cyto }}$ oligomerization and TssM $\mathrm{Cyto}-\mathrm{Tss}$ complex formation abolish T6SS sheath formation and function by impacting T6SS baseplate assembly and stability.

\section{DISCUSSION}

In this manuscript, we report the characterization of the cytoplasmic domain of the T6SS membrane core complex protein TssM from EAEC. We showed that TssM $\mathrm{Cyto}_{\text {C }}$ comprises two sub-domains, a domain resembling NTPases but lacking nucleotide binding and hydrolysis motifs, followed by a 110-amino-acid extension. Protein-protein interaction studies revealed that this extension mediates $\mathrm{Tss}_{\text {Cyto }}$ oligomerization and interaction with the TssG baseplate subunit. We finally defined specific motifs involved in these interactions and reported that these interactions are critical for the assembly of a functional T6SS. Models summarizing the findings reported in this study are depicted in Fig. 7.

We first defined the boundaries of the TssM trans-membrane segments using cysteine accessibility experiments. We determined that TssM is constituted of three TMH. The TssM $\mathrm{N}$-terminus locates in the cytoplasm and is followed by a trans-membrane hairpin, a cytoplasmic domain, and the third TMH, TMH3. Finally, the $\sim 750$-amino-acid C-terminal 
domain locates in the periplasm (Fig. 1C). This topology is similar to the topology of the Agrobacterium tumefaciens TssM protein previously defined using translational reporter fusions. ${ }^{29}$ Computer analyses of TssM proteins encoded within well-studied T6SS gene cluster showed that this topology is likely shared between all the homologues with the notable exception of the $P$. aeruginosa H1-T6SS TssM protein that is predicted to have a single TMH corresponding to TMH3 (Supp. Fig. S5).

The topology experiments also defined that TssM TMH2 and TMH3 delimit a 35-kDa cytoplasmic domain, TssM $\mathrm{Cyto}_{\text {. }}$ Our data showed that $\mathrm{TssM}_{\text {Cyto }}$ oligomerizes and interacts with TssL $L_{\text {Cyto }}$. The TssM $M_{\text {Cyto }}-T_{s s} M_{\text {Cyto }}$ and TssM $_{\text {Cyto }}-T_{s s} L_{\text {Cyto }}$ interactions have been reported in the Agrobacterium tumefaciens $\mathrm{T} 6 \mathrm{SS}^{29}$. The conservation of $\mathrm{TssM}_{\text {Cyto }}$ oligomerization and interaction with $\mathrm{TssL}_{\text {Cyto }}$ between A. tumefaciens and EAEC suggest that these contacts are important for T6SS function. Indeed, mutation of L279, a residue that participates to TssM $\mathrm{Cyto}_{\text {. }}$ oligomerization, severely impacts T6SS function in EAEC. The low-resolution of the recently published electron microscopy (EM) map of the 5-fold symmetry TssJLM MC does not allow to use docking simulations to precisely locate the TssM cytoplasmic domain and therefore to provide insight onto its oligomeric state in the complex. However, stoichiometry analyses and reconstruction of the MC suggested it is constituted of 5 dimers of TssJLM heterotrimers. ${ }^{16}$ These information suggest that, similarly to the TssL cytoplasmic domain ${ }^{32}$, TssM $\mathrm{M}_{\text {Cyto }}$ dimerizes. The biogenesis of the MC may therefore start with the formation of dimers of heterotrimers that will then symmetrize. Based on the TssM $\mathrm{M}_{\text {Cyto }}$ interaction with the TssL cytoplasmic domain, it has been proposed that these two domains form the large base of the T6SS MC. ${ }^{16}$ This cytoplasmic base corresponds to the docking site for the TssEFGK-VgrG baseplate complex. ${ }^{15,16,22}$ Indeed, our bacterial two-hybrid and co-immune precipitation experiments confirmed that $\mathrm{TssM}_{\text {Cyto }}$ interacts with two baseplate components, TssK and TssG (Fig. 7).

Bio-informatic analyses predict that $\mathrm{TssM}_{\text {Cyto }}$ comprises a N-terminal NTPase domain and a C-terminal DPY-30-like domain named TssM $\mathrm{Cyto}_{\mathrm{NTP}}$ and $\mathrm{TssM}_{\mathrm{Cyto} / \mathrm{Ct}}$, respectively.

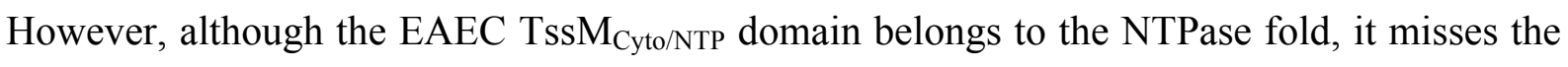
specific binding and hydrolysis motifs found in functional NTPases, such as the Walker A and $B$ motifs. Sequence alignment of TssM $\mathrm{Cyto}_{\mathrm{NTP}}$ domains from various bacterial species revealed that they categorize in two sub-families. While a number of TssM $\mathrm{Cyto}_{\mathrm{NTP}}$ domains do not carry these motifs, other TssM possess Walker A and B signatures, including that of $A$. 
tumefaciens, Edwardsiella tarda or P. aeruginosa H1-T6SS (Suppl. Fig. S2). Indeed, the detergent-solubilized A. tumefaciens TssM protein exhibits ATPase activity. ${ }^{37}$ However, mutations of these motifs in A.tumefaciens and E. tarda did not have the same impact on the function of the T6SS. Production of the K124A TssM variant in Edwardsiella was fully functional as shown by T6SS-dependent Hcp, VgrG and EvpP release. ${ }^{33}$ By contrast, ATP binding and hydrolysis regulate conformational changes within the periplasmic domain of the A. tumefaciens TssM protein and the Agrobacterium TssM K145A variant is unable to restore Hcp release. ${ }^{29,37}$ Therefore, $\mathrm{TssM}_{\mathrm{C} / \mathrm{NTP}}$ domains come in different flavours: active NTPase domains (e.g., A. tumefaciens), inactive NTPase domains (e.g., E. tarda) and NTPase fold lacking the functional motifs (e.g., EAEC). Interestingly, our protein-protein interaction studies showed that $\mathrm{TssM}_{\mathrm{C} / \mathrm{NTP}}$ contacts $\mathrm{TssK}$. In the case of TssM with functional NTP domains, it would be interesting to test whether the presence of TssK influences NTP binding and hydrolysis.

The TssM $\mathrm{M}_{\text {Cyto }} \mathrm{C}$-terminal extension shares structural homologies with the dimerization motif of DPY-30, a subunit of the histone methyltransferase complex in eukaryotic cells. This two- $\alpha$-helix motif forms an antiparallel bundle at the dimer interface, which is mediated by extensive hydrophobic and van der Waals interactions (Suppl. Fig. S4). ${ }^{42}$ Indeed, this DPY30-like hairpin and notably the conserved L279 residues are involved in TssM Cyto $_{\text {}}$ oligomerization. In addition to its role in $\mathrm{TssM}_{\text {Cyto }}$ oligomerization, this sub-domain is also required for proper interaction with the cytoplamic domain of TssL, TssL $\mathrm{Syto}_{\text {, }}$, and with TssG, one of the components of the T6SS baseplate (Fig. 7). Whereas we have not identified in this study the residues of $\mathrm{TssM}_{\mathrm{Cyto} / \mathrm{Ct}}$ mediating the interaction with $\mathrm{TssL}_{\mathrm{Cyto}}$, a conserved

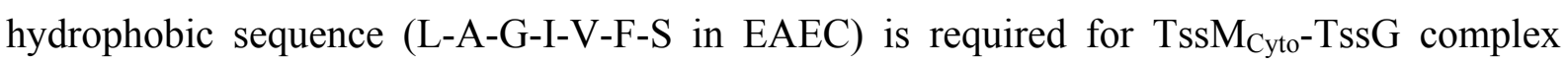
formation. Taken together, our results show that this relatively small sub-domain is responsible for several interactions. Interestingly, a similar case has been reported for DPY30 , which is a partner of several complexes involved in the regulation of chromatin and nucleosome organization. ${ }^{44}$ One may hypothesize that $\mathrm{TssM}_{\mathrm{Cyto} / \mathrm{Ct}}$ uses its DPY-30-like domain to interact sequentially with its different protein partners. Purification of the TssM $\mathrm{Cyto}^{-}$ TssL $L_{\text {Cyto }}$-TssG complex or high-resolution structure of the different binary complexes involving TssM $\mathrm{C}_{\mathrm{Cyto} / \mathrm{Ct}}$ will shed light on the dynamic nature of these interactions.

The interactions between TssM $\mathrm{Cyto}_{\text {and }}$ TssK and TssG, two components of the baseplate complex, might be important to recruit or to stabilize the $\mathrm{BC}$ at the cytoplasmic base 
of the TssJLM MC. In addition to this structural role, it is likely that these interactions are required for proper function of the baseplate. As shown for other contractile structures such as bacteriophages, the baseplate serves as assembly platform for the tail, but is also responsible for initiating sheath contraction. ${ }^{26,45-47}$ The TssM ${ }_{\mathrm{Cyto}}-\mathrm{Tss} \mathrm{G}$ interaction might be therefore important to regulate baseplate assembly, recruitment or sheath assembly and/or contraction. Indeed, point mutations disrupting the $\mathrm{TssM}_{\mathrm{Cyto}}-\mathrm{Tss} \mathrm{s}$ interaction destabilize the baseplate complex and prevent elongation of the tail sheath. It is interesting to note that TssM undergoes structural transitions ${ }^{37}$ and one may suggest that TssM conformational changes might be transduced to the baseplate via the TssM $\mathrm{Cyto} / \mathrm{NTP}-\mathrm{TssK}$ and/or $\mathrm{TssM}_{\mathrm{Cyto} / \mathrm{Ct}}-\mathrm{TssG}$ interactions leading to sheath assembly. As TssM has a large periplasmic domain and a short extension that lies outside of the cell ${ }^{16}$, it is a strong candidate to sense modifications of the cell envelope such as an attack by neighbouring cells, or contact with a prey, and to transmit the information to the baseplate complex. Further experiments will provide insights on how sheath assembly and/or contraction is regulated.

Our results also pointed that mutations disrupting $\mathrm{Tss}_{\mathrm{Cyto}}$ oligomerization and TssM $_{\text {Cyto }}$-TssG complex formation affect T6SS-dependent activities as they abolish TssBCsheath assembly and inhibit bacterial prey killing. The drastic effect of these mutations makes these interactions attractive targets for the rationale design of drugs that, by binding to TssM $_{\text {Cyto }}$, would hamper the T6SS activity and the delivery of harmful toxins. This approach has been successfully achieved in the case of the Brucella Type IV secretion VirB8 inner membrane subunit for which specific inhibitors of its dimerization were identified by a highthroughput bacterial two-hybrid screen and were further shown to inhibit Brucella infection of macrophages. ${ }^{48,49}$ This example emphasises the importance of understanding protein-protein interactions in bacterial secretion systems with the ultimate goal to target specific interactions with small molecule inhibitors.

\section{MATERials and Methods}

Bacterial strains, media, growth conditions and chemicals. Strains used in this study are listed in Supplementary Table S1. Escherichia coli K-12 DH5a, BTH101 and W3110 were used for cloning procedures, bacterial two-hybrid and co-immune precipitation, respectively. The E. coli K-12 W3110 strain carrying the pUA66-rrnB plasmid ( $g f p$ under control of the constitutive $r r n B$ ribosomal 
promoter, specifying strong and constitutive fluorescence, and kanamycin resistance ${ }^{50}$ was used as prey in antibacterial competition experiments. Enteroaggregative E. coli strain 17-2, and its $\Delta t s s M$, tssB-GFP and GFP-tssF derivatives ${ }^{15,24,30}$ were used in this study. Chromosomal fluorescent reporter insertions were obtained using the modified one-step inactivation procedure ${ }^{51}$ using the red recombinase expressed from $\mathrm{pKOBEG}^{52}$ as previously described ${ }^{28}$ using $\mathrm{pgfp}$-KD4 as template for Polymerase Chain Reaction (PCR) amplification. Briefly, the sfGFP-coding sequence and the kanamycin cassette were amplified from the $\mathrm{p} g f p$-KD4 vector $^{15}$ with oligonucleotides carrying 50 nucleotide extensions homologous to regions adjacent to the site of insertion. The PCR product was column purified (PCR and Gel Clean up kit, Promega) and electroporated. Kanamycin resistant clones were recovered and the insertion of the kanamycin cassette at the targeted site was verified by PCR. Kanamycin cassettes were then excised using pCP20.$^{51}$ tssM point mutations were engineered at the native locus on the chromosome by allelic replacement using the pKO3 suicide vector. ${ }^{53,54}$ Briefly, 172 tssB-GFP or GFP-tssF cells were transformed with a $\mathrm{pKO} 3$ plasmid in which a fragment of the $t s S M$ gene carrying the point mutations has been cloned (see below). Insertion of the plasmid into the chromosome was selected on chloramphenicol plates at $42^{\circ} \mathrm{C}$. Plasmid sequences removal was then selected on $5 \%$ sucrose plates without antibiotic and $t s s M$ point mutation recombinant strains were screened by PCR and confirmed by DNA sequencing (Eurofins,MWG). Unless specified, cells were grown in Luria broth (LB) or in sci-1 inducing medium (SIM: M9 minimal medium, glycerol 0.2\%, vitamin B1 $1 \mu \mathrm{g} / \mathrm{mL}$, casaminoacids $100 \mu \mathrm{g} / \mathrm{mL}$, LB $10 \%$, supplemented or not with bactoagar $1.5 \%)^{55}$ at $37^{\circ} \mathrm{C}$ with shaking. Plasmids were maintained by addition of ampicillin $(100 \mu \mathrm{g} / \mathrm{mL})$, kanamycin $(50 \mu \mathrm{g} / \mathrm{mL})$ or chloramphenicol $(30 \mu \mathrm{g} / \mathrm{mL})$. Gene expression from pASK-IBA37 and pBAD vectors was induced by the addition $0.1 \mu \mathrm{g} / \mathrm{mL}$ of anhydrotetracyclin (AHT, IBA Technology) and $0.02 \%$ of L-arabinose (Sigma-Aldrich), respectively.

Plasmid construction. Plasmids used in this study are listed in Supplementary Table S1. Polymerase Chain Reactions (PCR) were performed using a Biometra thermocycler using the Q5 high fidelity DNA polymerase (New England Biolabs). Restriction enzymes were purchased from New England Biolabs and used according the manufacturer's instructions. Custom oligonucleotides, listed in Supplementary Table S1, were synthesized by Sigma Aldrich. Enteroaggregative E. coli 17-2 chromosomal DNA was used as a template for all PCRs. E. coli strain DH5 $\alpha$ was used for cloning procedures. With the exception of the pKO3-'tss $M$ ' vector, plasmids have been constructed by restriction-free cloning ${ }^{56}$ as previously described ${ }^{30}$. Briefly, genes of interest were amplified with oligonucleotides introducing extensions annealing to the target vector. The double-stranded product of the first PCR was then been used as oligonucleotides for a second PCR using the target vector as template. pKO3-'tss $M^{\prime}$ has been constructed by the restriction-ligation procedure. A BamHI-SalI PCR product corresponding to a fragment of the $t s s$ gene (nucleotides 216-1569) was ligated into pKO3 digested by the same enzymes using T4 DNA ligase (New England Biolabs). Substitutions into 
pIBA37-FLAG TssM, pTssM Cyto $-\mathrm{T18}$, pT18-TssM ${ }_{\text {Cyto }}$ and pKO3-'tss $M$ have been introduced by sitedirected mutagenesis using complementary pairs of oligonucleotides and the Pfu Turbo high fidelity polymerase (Agilent Techologies). All constructs have been verified by restriction analysis and DNA sequencing (Eurofins, MWG).

Antibacterial assay. The antibacterial competition growth assay was performed as described. ${ }^{43}$ The wild-type E. coli strain W3110 bearing the $\mathrm{GFP}^{+}$kanamycin-resistant pUA66-rrnB plasmid $^{50}$ was used as prey in the competition assay. The kanamycin-resistant pUA66-rrnB plasmid provides a strong constitutive green fluorescent $\left(\mathrm{GFP}^{+}\right)$phenotype. Attacker and prey cells were grown for 16 hours in SIM, then diluted 100-fold in SIM. Once the culture reached an $\mathrm{OD}_{600}=0,8$, cells were harvested and resuspended to an $\mathrm{OD}_{600 \mathrm{~nm}}$ of 10 in SIM. Attacker and prey cells were mixed to a 4:1 ratio and 20- $\mu \mathrm{L}$ drops of the mixture were spotted in triplicate onto a pre-warmed dry SIM agar plate. After incubation for 4 hours at $37^{\circ} \mathrm{C}$, the bacterial spots were resuspended in $\mathrm{LB}$ and bacterial suspensions were normalized to an $\mathrm{OD}_{600}$ of 0.5 . For enumeration of viable prey cells, bacterial suspensions were serially diluted and spotted onto selective LB agar plates supplemented with kanamycin (for the $E$. coli prey cells). The experiments were done in triplicate, with identical results, and we report here the results of a representative experiment.

Substituted cysteine accessibility method (SCAM). Cysteine accessibility experiments were carried out as described ${ }^{57,58}$ with modifications..$^{30,31}$ A $40-\mathrm{mL}$ culture of strain $\Delta t s s M$ producing the TssM or cysteine-substituted TssM derivatives was induced for $t s s M$ gene expression with $0.02 \%$ AHT for 2 hours. Cells were harvested, resuspended in buffer A (100 mM HEPES (pH 7.5), $250 \mathrm{mM}$ sucrose, 25 $\mathrm{mM} \mathrm{MgCl} 2,0.1 \mathrm{mM} \mathrm{KCl}$ ) to a final $\mathrm{OD}_{600}$ of 12 in $500 \mu \mathrm{L}$ of buffer A. MPB (Molecular Probes) was added to a final concentration of $100 \mu \mathrm{M}$ (from a $20 \mathrm{mM}$ stock freshly dissolved in DMSO) and the cells were incubated for $30 \mathrm{~min}$ at $25^{\circ} \mathrm{C}$. $\beta$-Mercaptoethanol ( $20 \mathrm{mM}$ final concentration) was added to quench the biotinylation reaction, and cells were washed twice in buffer A, and resuspended in buffer A containing $N$-ethyl maleimide (final concentration $5 \mathrm{mM}$ ) to block all free sulfhydryl residues. After incubation $20 \mathrm{~min}$ at $25^{\circ} \mathrm{C}$, cells were disrupted by four passages at the French press at 800 psi. Membranes recovered by ultracentrifugation $40 \mathrm{~min}$ at $100000 \times \mathrm{g}$ were resuspended in $1 \mathrm{~mL}$ of buffer B (10 mM Tris (pH 8.0), $100 \mathrm{mM} \mathrm{NaCl,} \mathrm{1 \% (w/v)} \mathrm{Triton} \mathrm{X-100,} \mathrm{protease} \mathrm{inhibitor} \mathrm{cocktail}$ (Complete, Roche)). After incubation on a wheel for 2 hours, unsolubilized material was removed by centrifugation $15 \mathrm{~min}$ at $20,000 \times g$, and solubilized proteins were subjected to immune precipitations using anti-FLAG M2 affinity gel (Sigma-Aldrich). After 3 hours of incubation on a wheel, the beads were washed twice with $1 \mathrm{~mL}$ buffer $\mathrm{B}$, and once with buffer $\mathrm{C}(10 \mathrm{mM}$ Tris $(\mathrm{pH} 8.0), 100 \mathrm{mM} \mathrm{NaCl}$, $0.1 \%(\mathrm{w} / \mathrm{v})$ Triton X-100). Beads were air-dried, resuspended in Laemmli buffer and subjected to SDS-PAGE and immunodetection with anti-FLAG antibodies, and streptavidin coupled to alkaline phosphatase. 
Bacterial two-hybrid assay. The adenylate cyclase-based bacterial two-hybrid technique ${ }^{59}$ was used as previously published. ${ }^{24,60}$ Briefly, pairs of proteins to be tested were fused to the isolated T18 and T25 catalytic domains of the Bordetella adelynate cyclase. After transformation of the two plasmids producing the fusion proteins into the reporter BTH101 strain, plates were incubated at $30^{\circ} \mathrm{C}$ for 48 hours. Three independent colonies for each transformation were inoculated into $600 \mu \mathrm{L}$ of LB medium supplemented with ampicillin, kanamycin and IPTG $(0.5 \mathrm{mM})$. After overnight growth at $30^{\circ} \mathrm{C}, 10 \mu \mathrm{L}$ of each culture were dropped onto LB supplemented with $40 \mu \mathrm{g} / \mathrm{mL}^{-1}$ bromo-chloro-indolyl- $\beta$-Dgalactopyrannoside (X-Gal) and incubated for 16 hours at $30^{\circ} \mathrm{C}$. The experiments were done at least in triplicate and a representative result is shown.

Co-immune precipitation. $100 \mathrm{~mL}$ of $\mathrm{W} 3110$ cells producing proteins of interest were grown to an $\mathrm{OD}_{600}$ of 0.4 and the expression of the cloned genes were induced with AHT or L-arabinose for 45 min. The cells were harvested, and the pellets were resuspended in Tris- $\mathrm{HCl} 20 \mathrm{mM} \mathrm{pH} \mathrm{8.0,} \mathrm{NaCl}$ $100 \mathrm{mM}$, sucrose 30\%, EDTA $1 \mathrm{mM}$, lysozyme $100 \mu \mathrm{g} / \mathrm{mL}$, DNase $100 \mu \mathrm{g} / \mathrm{mL}$, RNase $100 \mu \mathrm{g} / \mathrm{mL}$ supplemented with protease inhibitors (Complete, Roche) to an $\mathrm{OD}_{600}$ of 80 and incubated on ice for $20 \mathrm{~min}$. Cells were lysed by three passages at the French Press (800 psi) and lysates were clarified by centrifugation at $20,000 \times g$ for $20 \mathrm{~min}$. Supernatants were used for co-immune precipitation using anti-FLAG M2 affinity gel (Sigma-Aldrich). After 3 hours of incubation, the beads were washed three times with $1 \mathrm{~mL}$ of Tris- $\mathrm{HCl} 20 \mathrm{mM} \mathrm{pH} \mathrm{8.0,} \mathrm{NaCl} 100 \mathrm{mM}$, sucrose $15 \%$, resuspended in $25 \mu \mathrm{L}$ of Laemmli loading buffer, boiled for $10 \mathrm{~min}$ and subjected to SDS-PAGE and immunodetection analyses.

Fluorescence microscopy and statistical analyses. Overnight cultures of entero-aggregative E. coli 17-2 derivatives strains were diluted 1:100 in SIM medium and grown for 6 hours to an $\mathrm{OD}_{600} \sim 1.0$ to maximize expression of the $s c i-1$ T6SS gene cluster. ${ }^{55}$ Cells were washed in phosphate buffered saline (PBS), resuspended in PBS to an $\mathrm{OD}_{600} \sim 50$ and spotted on a thin pad of $1.5 \%$ agarose in PBS and covered with a cover slip. Microscopy recording and digital image processing have been performed as previously described. ${ }^{15,16,18,20,24}$ The $\mathrm{Z}$ project (average intensity) plugin has been used to merge and flatten all $\mathrm{Z}$ planes. Microscopy analyses were performed at least six times, each with technical triplicate, and a representative experiment is shown. The number of sheath per number of cells and sfGFP-TssF foci was measured manually.

Computer analyses. Trans-membrane helix predictions were made using HMMTop ${ }^{61}$, TMHMM $^{62}$, TMpred $^{63}$ and PHDhtm ${ }^{64}$. Secondary structure predictions were made using the Psipred server (http://bioinf.cs.ucl.ac.uk/psipred/). Structural predictions and homology modelling of the tridimensional structure of $\mathrm{TssM}_{\mathrm{Cyto} / \mathrm{NTP}}$ and $\mathrm{TssM}_{\mathrm{Cyto} / \mathrm{Ct}}$ were performed using HHpred ${ }^{39}$ or SwissModel $^{65}$, respectively. Figures were made using Chimera ${ }^{66}$. Amino-acid sequences were aligned with 
$\mathrm{T}_{-} \mathrm{COFFEE}^{67}$ and phylogenetic analyses were performed with phylogeny.fr ${ }^{68}$.

Miscellaneous. SDS-polyacrylamide gel electrophoresis was performed using standard protocols. For immunostaining, proteins were transferred onto nitrocellulose membranes, and immunoblots were probed with primary antibodies, and goat secondary antibodies coupled to alkaline phosphatase, and developed in alkaline buffer in presence of 5-bromo-4-chloro-3-indolylphosphate and nitroblue tetrazolium. The anti-FLAG (M2 clone, Sigma-Aldrich), anti-VSV-G (clone P5D4, Sigma-Aldrich) monoclonal antibodies, the alkaline phosphatase-conjugated streptavidin (Pierce) and alkaline phosphatase-conjugated goat anti-mouse secondary antibodies (Beckman Coulter) have been purchased as indicated and used as recommended by the manufacturer.

\section{ACKNOWLEDGEMENTS}

We thank the members of the Cascales, Lloubès, Cambillau, Sturgis and Bouveret research groups for helpful discussions, Laure Journet for critical reading of the manuscript, Emmanuelle Bouveret and Julie Viala for advices and protocols for pKO3-dependent chromosomal engineering, Abdlerahim Zoued for statistical analyses, Olivier Uderso, Isabelle Bringer and Annick Brun for technical assistance, and Jimmy Switrumpey for encouragements. This work was supported by the Centre National de la Recherche Scientifique, the Aix-Marseille Université, and grants from the Agence Nationale de la Recherche to E.C. (ANR-10-JCJC-1303-03 and ANR-14-CE14-0006-02) and from the European Society of Clinical Microbiology and Infectious Diseases (ESCMID) to E.D. L.L. is supported by a doctoral fellowship from the French Ministry of Research and a end-of-thesis fellowship from the Fondation pour la Recherche Médicale (FDT20160435498). E.D. was supported by an EMBO short-term fellowship (ASTF-417-2015).

\section{REFERENCES}

1. Russell, A. B., Peterson, S. B. \& Mougous, J. D. (2014). Type VI secretion system effectors: poisons with a purpose. Nat Rev Microbiol. 12, 137-148.

2. Durand, E., Cambillau, C., Cascales, E. \& Journet, L. (2014). VgrG, Tae, Tle, and beyond: the versatile arsenal of Type VI secretion effectors. Trends Microbiol. 22, 498-507.

3. Alcoforado Diniz, J., Liu, Y. C. \& Coulthurst, S. J. (2015). Molecular weaponry: diverse effectors delivered by the Type VI secretion system. Cell Microbiol. 17, 1742-1751.

4. Hachani, A., Wood, T. E. \& Filloux, A. (2015). Type VI secretion and anti-host effectors. Curr Opin Microbiol. 29, 81-93. 
5. Bönemann, G., Pietrosiuk, A. \& Mogk, A. (2010). Tubules and donuts: a type VI secretion story. Mol Microbiol. 76, 815-821.

6. Cascales, E. \& Cambillau, C. (2012). Structural biology of type VI secretion systems. Philos Trans R Soc Lond B Biol Sci. 367, 1102-1111.

7. Coulthurst, S. J. (2013). The Type VI secretion system - a widespread and versatile cell targeting system. Res Microbiol.164, 640-654.

8. Zoued, A., Brunet, Y. R., Durand, E., Aschtgen, M. S., Logger, L., Douzi, B., Journet, L., Cambillau, C. \& Cascales, E. (2014). Architecture and assembly of the Type VI secretion system. Biochim Biophys Acta. 1843, 1664-1673.

9. Kube, S. \& Wendler, P. (2015). Structural comparison of contractile nanomachines. AIMS Biophysics. 2, 88-115.

10. Basler, M. (2015). Type VI secretion system: secretion by a contractile nanomachine. Philos Trans R Soc Lond B Biol Sci. 370, 1679.

11. Ho, B. T., Dong, T. G. \& Mekalanos, J. J. (2014). A view to a kill: the bacterial type VI secretion system. Cell Host Microbe. 15, 9-21.

12. Cianfanelli, F. R., Monlezun, L. \& Coulthurst, S. J. (2016). Aim, load, fire: the Type VI secretion system, a bacterial nanoweapon. Trends Microbiol. 24, 51-62.

13. Leiman, P. G., Basler, M., Ramagopal, U. A., Bonanno, J. B., Sauder, J. M., Pukatzki, S., Burley, S. K., Almo, S. C. \& Mekalanos, J. J. (2009). Type VI secretion apparatus and phage tailassociated protein complexes share a common evolutionary origin. Proc Natl Acad Sci USA. 106, 4154-4159.

14. Basler, M., Pilhofer, M., Henderson, G. P., Jensen, G. J. \& Mekalanos, J. J. (2012). Type VI secretion requires a dynamic contractile phage tail-like structure. Nature. 483, 182-186.

15. Brunet, Y. R., Zoued, A., Boyer, F., Douzi, B. \& Cascales, E. (2015). The Type VI secretion TssEFGK-VgrG phage-like baseplate is recruited to the TssJLM membrane complex via multiple contacts and serves as assembly platform for tail tube/sheath polymerization. PLoS Genet. 11, e1005545.

16. Durand, E., Nguyen, V. S., Zoued, A., Logger, L., Péhau-Arnaudet, G., Aschtgen, M. S., Spinelli, S., Desmyter, A., Bardiaux, B., Dujeancourt, A., Roussel, A., Cambillau, C., Cascales, E. \& Fronzes, R. (2015). Biogenesis and structure of a type VI secretion membrane core complex. Nature. 523, 555-560.

17. Ballister, E. R., Lai, A. H., Zuckermann, R. N., Cheng, Y. \& Mougous, J. D. (2008). In vitro selfassembly of tailorable nanotubes from a simple protein building block. Proc Natl Acad Sci USA. $105,3733-3738$.

18. Brunet, Y. R., Hénin, J., Celia, H. \& Cascales, E. (2014). Type VI secretion and bacteriophage tail tubes share a common assembly pathway. EMBO Rep. 15, 315-321.

19. Kudryashev, M., Wang, R. Y., Brackmann, M., Scherer, S., Maier, T., Baker, D., DiMaio, F., Stahlberg, H., Egelman, E. H. \& Basler, M. (2015). Structure of the type VI secretion system contractile sheath. Cell. 160, 952-962. 
20. Brunet, Y. R., Espinosa, L., Harchouni, S., Mignot, T. \& Cascales, E. (2013). Imaging type VI secretion-mediated bacterial killing. Cell Rep. 3, 36-41.

21. Basler, M., Ho, B. T. \& Mekalanos, J. J. (2013). Tit-for-tat: type VI secretion system counterattack during bacterial cell-cell interactions. Cell. 152, 884-894.

22. Zoued, A., Durand, E., Brunet, Y. R., Spinelli, S., Douzi, B., Guzzo, M., Flaugnatti, N., Legrand, P., Journet, L., Fronzes, R., Mignot, T., Cambillau, C. \& Cascales, E. (2016). Priming and polymerization of a bacterial contractile tail structure. Nature. 531, 59-63.

23. Pukatzki, S., Ma, A. T., Revel, A. T., Sturtevant, D. \& Mekalanos, J. J. (2007). Type VI secretion system translocates a phage tail spike-like protein into target cells where it cross-links actin. Proc Natl Acad Sci USA. 104, 15508-15513.

24. Zoued, A., Durand, E., Bebeacua, C., Brunet, Y. R., Douzi, B., Cambillau, C., Cascales, E. \& Journet, L. (2013). TssK is a trimeric cytoplasmic protein interacting with components of both phage-like and membrane anchoring complexes of the type VI secretion system. J Biol Chem. 288, 27031-27041.

25. English, G., Byron, O., Cianfanelli, F. R., Prescott, A. R. \& Coulthurst, S. J. (2014). Biochemical analysis of TssK, a core component of the bacterial Type VI secretion system, reveals distinct oligomeric states of TssK and identifies a TssK-TssFG subcomplex. Biochem J. 461, 291-304.

26. Taylor, N. M., Prokhorov, N. S., Guerrero-Ferreira, R. C., Shneider, M. M., Browning, C., Goldie, K. N., Stahlberg, H. \& Leiman, P. G. (2016). Structure of the T4 baseplate and its function in triggering sheath contraction. Nature. 533, 346-352.

27. Planamente, S., Salih, O., Manoli, E., Albesa-Jové, D., Freemont, P. S. \& Filloux, A. (2016). TssA forms a gp6-like ring attached to the type VI secretion sheath. EMBO J. in press.

28. Aschtgen, M. S., Bernard, C. S., De Bentzmann, S., Lloubès, R. \& Cascales, E. (2008). SciN is an outer membrane lipoprotein required for type VI secretion in enteroaggregative Escherichia coli. J Bacteriol. 190, 7523-7531.

29. Ma, L. S., Lin, J. S. \& Lai, E. M. (2009). An IcmF family protein, ImpLM, is an integral inner membrane protein interacting with ImpKL, and its walker a motif is required for type VI secretion system-mediated Hcp secretion in Agrobacterium tumefaciens. J Bacteriol. 191, 43164129.

30. Aschtgen, M. S., Gavioli, M., Dessen, A., Lloubès, R. \& Cascales, E. (2010). The SciZ protein anchors the enteroaggregative Escherichia coli Type VI secretion system to the cell wall. Mol Microbiol. 75, 886-899.

31. Aschtgen, M. S., Zoued, A., Lloubès, R., Journet, L. \& Cascales, E. (2012). The C-tail anchored TssL subunit, an essential protein of the enteroaggregative Escherichia coli Sci-1 Type VI secretion system, is inserted by YidC. Microbiologyopen. 1, 71-82.

32. Durand, E., Zoued, A., Spinelli, S., Watson, P. J., Aschtgen, M. S., Journet, L., Cambillau, C. \& Cascales, E. (2012). Structural characterization and oligomerization of the TssL protein, a component shared by bacterial type VI and type IVb secretion systems. J Biol Chem. 287, 1415714168.

33. Zheng, J. \& Leung, K. Y. (2007). Dissection of a type VI secretion system in Edwardsiella tarda. 
Mol Microbiol. 66, 1192-1206.

34. Felisberto-Rodrigues, C., Durand, E., Aschtgen, M. S., Blangy, S., Ortiz-Lombardia, M., Douzi, B., Cambillau, C. \& Cascales, E. (2011). Towards a structural comprehension of bacterial type VI secretion systems: characterization of the TssJ-TssM complex of an Escherichia coli pathovar. PLoS Pathog. 7, e1002386.

35. Nguyen, V. S., Logger, L., Spinelli, S., Desmyter, A., Le, T. T., Kellenberger, C., Douzi, B., Durand, E., Roussel, A., Cascales, E. \& Cambillau, C. (2015). Inhibition of type VI secretion by an anti-TssM llama nanobody. PLoS One. 10, e0122187.

36. Gerc, A. J., Diepold, A., Trunk, K., Porter, M., Rickman, C., Armitage, J. P., Stanley-Wall, N. R. \& Coulthurst, S. J. (2015). Visualization of the Serratia Type VI secretion system reveals unprovoked attacks and dynamic assembly. Cell Rep. 12, 2131-2142.

37. Ma, L. S., Narberhaus, F. \& Lai, E. M. (2012). IcmF family protein TssM exhibits ATPase activity and energizes type VI secretion. J Biol Chem. 287, 15610-15621.

38. Bogdanov, M., Zhang, W., Xie, J. \& Dowhan, W. (2005). Transmembrane protein topology mapping by the substituted cysteine accessibility method (SCAM(TM)): application to lipidspecific membrane protein topogenesis. Methods. 36, 148-171.

39. Söding, J., Biegert, A. \& Lupas, A. N. (2005). The HHpred interactive server for protein homology detection and structure prediction. Nucleic Acids Res. 33, W244-248.

40. Baugh, L., Gallagher, L. A., Patrapuvich, R., Clifton, M. C., Gardberg, A. S., Edwards, T. E., Armour, B., Begley, D. W., Dieterich, S. H., Dranow, D. M., Abendroth, J., Fairman, J. W., Fox, D. 3rd, Staker, B. L., Phan, I., Gillespie, A., Choi, R., Nakazawa-Hewitt, S., Nguyen, M. T., Napuli, A., Barrett, L., Buchko, G. W., Stacy, R., Myler, P. J., Stewart, L. J., Manoil, C. \& Van Voorhis, W. C. (2013). Combining functional and structural genomics to sample the essential Burkholderia structome. PLoS One. 8, e53851.

41. Hanukoglu, I. (2015). Rossmann fold: A beta-alpha-beta fold at dinucleotide binding sites. Biochem Mol Biol Educ. 43, 206-209.

42. Wang, X., Lou, Z., Dong, X., Yang, W., Peng, Y., Yin, B., Gong, Y., Yuan, J., Zhou, W., Bartlam, M., Peng, X. \& Rao, Z. (2009). Crystal structure of the C-terminal domain of human DPY-30-like protein: A component of the histone methyltransferase complex. J Mol Biol. 390, 530-537.

43. Flaugnatti, N., Le, T. T., Canaan, S., Aschtgen, M. S., Nguyen, V. S., Blangy, S., Kellenberger, C., Roussel, A., Cambillau, C., Cascales, E. \& Journet, L. (2016). A phospholipase A1 anti-bacterial T6SS effector interacts directly with the C-terminal domain of the $\mathrm{VgrG}$ spike protein for delivery. Mol Microbiol. 99, 1099-1118.

44. Tremblay, V., Zhang, P., Chaturvedi, C. P., Thornton, J., Brunzelle, J. S., Skiniotis, G., Shilatifard, A., Brand, M. \& Couture, J. F. (2014). Molecular basis for DPY-30 association to COMPASSlike and NURF complexes. Structure. 22, 1821-1830.

45. Kostyuchenko, V. A., Leiman, P. G., Chipman, P. R., Kanamaru, S., van Raaij, M. J., Arisaka, F., Mesyanzhinov, V. V. \& Rossmann, M. G. (2003). Three-dimensional structure of bacteriophage T4 baseplate. Nat Struct Biol. 10, 688-693.

46. Kostyuchenko, V. A., Chipman, P. R., Leiman, P. G., Arisaka, F., Mesyanzhinov, V. V. \& 
Rossmann, M. G. (2005). The tail structure of bacteriophage T4 and its mechanism of contraction. Nat Struct Mol Biol. 12, 810-813.

47. Leiman, P. G., Arisaka, F., van Raaij, M. J., Kostyuchenko, V. A., Aksyuk, A. A., Kanamaru, S. \& Rossmann, M. G. (2010). Morphogenesis of the T4 tail and tail fibers. Virol J. 7, 355.

48. Paschos, A., den Hartigh, A., Smith, M. A., Atluri, V. L., Sivanesan, D., Tsolis, R. M. \& Baron, C. (2011). An in vivo high-throughput screening approach targeting the type IV secretion system component VirB8 identified inhibitors of Brucella abortus 2308 proliferation. Infect Immun. 79, 1033-1043.

49. Smith, M. A., Coinçon, M., Paschos, A., Jolicoeur, B., Lavallée, P., Sygusch, J. \& Baron, C. (2012). Identification of the binding site of Brucella VirB8 interaction inhibitors. Chem Biol. 19, 1041-1048.

50. Zaslaver, A., Bren, A., Ronen, M., Itzkovitz, S., Kikoin, I., Shavit, S., Liebermeister, W., Surette, M. G. \& Alon, U. (2006). A comprehensive library of fluorescent transcriptional reporters for Escherichia coli. Nat Methods. 3, 623-628.

51. Datsenko, K. A. \& Wanner, B. L. (2000). One-step inactivation of chromosomal genes in Escherichia coli K-12 using PCR products. Proc Natl Acad Sci USA. 97, 6640-6645.

52. Chaveroche, M. K., Ghigo, J. M. \& d'Enfert, C. (2000). A rapid method for efficient gene replacement in the filamentous fungus Aspergillus nidulans. Nucleic Acids Res. 28, E97.

53. Link, A. J., Phillips, D. \& Church, G. M. (1997). Methods for generating precise deletions and insertions in the genome of wild-type Escherichia coli: application to open reading frame characterization. J Bacteriol. 179, 6228-6237.

54. Battesti, A. \& Bouveret, E. (2006). Acyl carrier protein/SpoT interaction, the switch linking SpoTdependent stress response to fatty acid metabolism. Mol Microbiol. 62, 1048-1063.

55. Brunet, Y. R., Bernard, C. S., Gavioli, M., Lloubès, R. \& Cascales, E. (2011). An epigenetic switch involving overlapping fur and DNA methylation optimizes expression of a type VI secretion gene cluster. PLoS Genet. 7, e1002205.

56. van den Ent, F. \& Löwe, J. (2006). RF cloning: a restriction-free method for inserting target genes into plasmids. J Biochem Biophys Methods. 67, 67-74.

57. Jakubowski, S. J., Krishnamoorthy, V., Cascales, E. \& Christie, P. J. (2004). Agrobacterium tumefaciens VirB6 domains direct the ordered export of a DNA substrate through a type IV secretion system. J Mol Biol. 341, 961-977.

58. Goemaere, E. L., Devert, A., Lloubès, R. \& Cascales, E. (2007). Movements of the TolR Cterminal domain depend on TolQR ionizable key residues and regulate activity of the Tol complex. J Biol Chem. 282, 17749-17757.

59. Karimova, G., Pidoux, J., Ullmann, A. \& Ladant, D. (1998). A bacterial two-hybrid system based on a reconstituted signal transduction pathway. Proc Natl Acad Sci USA. 95, 5752-5756.

60. Battesti, A. \& Bouveret, E. (2012). The bacterial two-hybrid system based on adenylate cyclase reconstitution in Escherichia coli. Methods. 58, 325-334. 
61. Tusnády, G. E. \& Simon, I. (1998). Principles governing amino acid composition of integral membrane proteins: application to topology prediction. J Mol Biol. 283, 489-506.

62. Krogh, A., Larsson, B., von Heijne, G. \& Sonnhammer, E. L. (2001). Predicting transmembrane protein topology with a hidden Markov model: application to complete genomes. J Mol Biol. 305, 567-580.

63. Hofmann, K. \& Stoffel, W. (1993). A database of membrane spanning protein segments. Biol. Chem. 374, 166.

64. Rost, B., Fariselli, P. \& Casadio, R. (1996). Topology prediction for helical transmembrane proteins at $86 \%$ accuracy. Protein Sci. 5, 1704-1718.

65. Biasini, M., Bienert, S., Waterhouse, A., Arnold, K., Studer, G., Schmidt, T., Kiefer, F., Gallo Cassarino, T., Bertoni, M., Bordoli, L. \& Schwede, T. (2014). SWISS-MODEL: modelling protein tertiary and quaternary structure using evolutionary information. Nucleic Acids Res. 42, W252-258.

66. Pettersen, E. F., Goddard, T. D., Huang, C. C., Couch, G. S., Greenblatt, D. M., Meng, E. C. \& Ferrin, T. E. (2004). UCSF Chimera - a visualization system for exploratory research and analysis. J Comput Chem. 25, 1605-1612.

67. Di Tommaso, P., Moretti, S., Xenarios, I., Orobitg, M., Montanyola, A., Chang, J. M., Taly, J. F. \& Notredame, C. (2011). T-Coffee: a web server for the multiple sequence alignment of protein and RNA sequences using structural information and homology extension. Nucleic Acids Res. 39, W13-17.

68. Dereeper, A., Guignon, V., Blanc, G., Audic, S., Buffet, S., Chevenet, F., Dufayard, J. F., Guindon, S., Lefort, V., Lescot, M., Claverie, J. M. \& Gascuel, O. (2008). Phylogeny.fr: robust phylogenetic analysis for the non-specialist. Nucleic Acids Res. 36, W465-469.

69. Zoued, A., Cassaro, C.J., Durand, E., Douzi, B., España, A.P., Cambillau, C., Journet, L. \& Cascales, E. (2016). Structure-function analysis of the TssL cytoplasmic domain reveals a new interaction between the Type VI secretion baseplate and membrane complexes. J Mol Biol.

\section{LEGEND TO FIGURES}

\section{Figure 1. TssM is a polytopic inner membrane protein.}

A. The trans-membrane helices predicted using the algorithms listed on the left are represented by black rectangles. The EAEC Sci-1 TssM natural cysteine residues, as well as the cysteine substitutions engineered in this study are indicated. Filled circles indicate cysteine residues labelled with the 3-(Nmaleimidylpropionyl) biocytin (MPB) probe, whereas open circles indicate unlabelled cysteine residues. Arrowheads indicate trans-membrane segments determined experimentally. B. Accessibility of cysteine residues. Whole EAEC $\Delta t s s M$ cells producing wild-type TssM (WT) or the indicated mutant proteins were labelled with the MPB probe, lysed, solubilized, and TssM and mutant proteins were immunoprecipitated with anti-FLAG-coupled beads. The precipitated material was subjected to 
SDS-PAGE and Western blot analysis using anti-FLAG antibody (TssM detection, upper panel) and streptavidin coupled to alkaline phosphatase (MPB-labelled TssM detection, lower panel). Molecular weight markers are indicated on the left. C. Topology model for the EAEC TssM protein at the inner membrane. The localizations of the labelled and unlabelled cysteine residues are indicated by filled and open circles respectively. The cytoplasmic $\left(\mathrm{TssM}_{\mathrm{Cyto}}\right)$ and periplasmic $\left(\mathrm{TssM}_{\mathrm{Peri}}\right)$ domains are highlighted in green and orange respectively. The three trans-membrane segments identified by the accessibility studies are shown, with their membrane boundaries (in blue).

\section{Figure 2. TssM $M_{\text {Cyto }}$ interaction network identified by bacterial two-hybrid analysis.}

BTH101 reporter cells carrying pairs of plasmids producing the indicated T6SS proteins fused to the T18 or T25 domain of the Bordetella adenylate cyclase were spotted on X-Gal-IPTG indicator LB agar plates. Only the cytoplasmic (Cyto) or periplasmic (Peri) domains were used for membraneanchored proteins. Controls include T18 and T25 fusions to TolB and Pal, two proteins that interact but unrelated to the T6SS.

\section{Figure 3. Structural architecture of Tss $M_{\text {Cyto. }}$.}

A. The cytoplasmic domain of TssM, TssM $\mathrm{Cyto}_{\text {, }}$, delimitated by the trans-membrane helices 2 and 3 (TMH2 and TMH3 respectively) could be partitioned into an NTPase-like domain (NTP, blue) and a C-terminal extension ( $\mathrm{Ct}$, green).TssM $\mathrm{Cyto}_{\mathrm{NTP}}$ was modelled using HHpred based on the X-ray structure of the Burkholderia thailandensis EngB GTP-binding protein (PDB ID: 4DHE). TssM $\mathrm{M}_{\mathrm{Cyt} / \mathrm{Ct}}$ was modelled using SwissModel based on the X-ray structure of the C-terminal domain of the human DPY-30-like protein, a component of the histone methyltransferase complex (PDB ID: 3G36). All images were made with Chimera. ${ }^{66}$

Figure 4. The two TssM $M_{\text {Cyto }}$ sub-domains mediate interaction with two baseplate components.

A. Bacterial two-hybrid assay. BTH101 reporter cells carrying pairs of plasmids producing the indicated T6SS proteins fused to the T18 or T25 domain of the Bordetella adenylate cyclase were

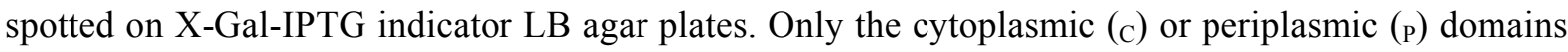
were used for membrane-anchored proteins. Controls include T18 and T25 fusions to TolB and Pal, two proteins that interact but unrelated to the T6SS. B. Co-immunoprecipitation assay. Soluble lysates from $5 \times 10^{10}$ E. coli $\mathrm{K} 12 \mathrm{~W} 3110$ cells producing FLAG-tagged $\mathrm{TssM}_{\mathrm{Cyto}}\left(\mathrm{M}_{\text {Cyto_fL }}\right)$, FLAG-tagged $\mathrm{TssM}_{\mathrm{Cyto} / \mathrm{NTP}}\left(\mathrm{NTP}_{\mathrm{FL}}\right)$ or FLAG-tagged TssM $\mathrm{Cyto}_{\mathrm{Ct}}\left(\mathrm{Ct}_{\mathrm{FL}}\right)$ and VSV-G-tagged TssG $\left(\mathrm{TssG}_{\mathrm{V}}\right)$ or TssK $\left(\mathrm{TssK}_{\mathrm{V}}\right)$ proteins were subjected to immunoprecipitation with anti-FLAG-coupled beads. The total soluble (Tot) and the immunoprecipitated (IP) material were separated by $12.5 \%$ acrylamide SDSPAGE and immunodetected with anti-VSVG $\left(\mathrm{TssG}_{\mathrm{V}}\right.$ or $\left.\mathrm{TssK}_{\mathrm{V}}\right)$ monoclonal antibodies. Molecular weight markers (in $\mathrm{kDa}$ ) are indicated on the left. The asterisk on the lower panel indicates a degradation product from the TssK protein. 


\section{Figure 5. Specific conserved motifs mediate Tss $_{\text {Cyto }}$ dimerization and interaction with TssG}

A. Bacterial two-hybrid assay. BTH101 reporter cells carrying pairs of plasmids producing the indicated T6SS proteins fused to the T18 or T25 domain of the Bordetella adenylate cyclase were spotted on X-Gal-IPTG indicator LB agar plates. Only the cytoplasmic (c) or periplasmic (P) domains were used for membrane-anchored proteins. Controls include T18 and T25 fusions to TolB and Pal, two proteins that interact but unrelated to the T6SS. B. Co-immunoprecipitation assay. Soluble lysates from $5 \times 10^{10}$ E. coli $\mathrm{K} 12 \mathrm{~W} 3110$ cells producing FLAG-tagged $\mathrm{TssM}_{\mathrm{C}} \mathrm{WT}\left(\mathrm{M}_{\mathrm{Cyt} \_ \text {FL }}\right)$ or mutants and VSV-G-tagged TssG $\left(\mathrm{TssG}_{\mathrm{V}}\right)$ or $\mathrm{TssM}_{\text {Cyto }} \quad\left(\mathrm{TssM}_{\text {Cyto_v }}\right)$ proteins were subjected to immunoprecipitation with anti-FLAG-coupled beads. The total soluble (Tot) and the immunoprecipitated (IP) material were separated by $12.5 \%$ acrylamide SDS-PAGE and immunodetected with anti-FLAG (lower panels) and anti-VSV-G (upper panels) monoclonal antibodies. Molecular weight markers (in $\mathrm{kDa}$ ) are indicated on the left.

Figure 6. TssM $M_{\text {Cyto }}$ oligomerization and interaction with the TssG baseplate are essential for T6SS function, sheath assembly and baseplate stability

A. Anti-bacterial assay. E. coli $\mathrm{K}-12$ prey cells (W3110 $g f p^{+}, \mathrm{kan}^{\mathrm{R}}$ ) were mixed with the indicated attacker cells, spotted onto Sci-1 inducing medium (SIM) agar plates and incubated for 4 hours at $37^{\circ} \mathrm{C}$. The number of recovered $E$. coli prey cells is indicated in the graph (in $\log 10$ of colony-forming unit (cfu)). The circles indicate values from three independent assays, and the average is indicated by the bar. B. Image recordings of the TssB-sfGFP fusion protein in the indicated cells. Statistical analyses ( $n=$ number of sheath/ cell) are indicated under each strain. The number of cells studied per strain $(n)$ is 150 . The scale bare is $2 \mu \mathrm{m}$. C. Image recordings of the sfGFP-TssF fusion protein in the indicated cells. The scale bare is $2 \mu \mathrm{m}$. D. Statistical analyses of sfGFP-TssF in the indicated strains. Shown are box-and-whisker plots of the measured number of sfGFP-TssF foci per cell for each strain with the lower and upper boundaries of the boxes corresponding the $25 \%$ and $75 \%$ percentiles respectively. The black bold horizontal bar represents the median values for each strain and the whiskers represent the $10 \%$ and $90 \%$ percentiles. Outliers are shown as open circle. $n$ indicates the number of cells analyzed per strain.

Figure 7. Schematic representation of the $\operatorname{TssM}_{\mathrm{Cyto}}$ interaction network. A. Schematic representation of the TssJLM membrane complex (MC) and its interactions with the TssKEFG-VgrG baseplate complex (BC). The TssM $\mathrm{M}_{\mathrm{Cyto} / \mathrm{NTP}}$ and $\mathrm{Tss} \mathrm{M}_{\mathrm{Cyto} / \mathrm{Ct}}$ and the TssL cytoplasmic domain (TssL $\mathrm{L}_{\mathrm{Cyto}}$ ) are shown in blue, green and orange respectively. The interactions defined in this study are indicated by red arrows. Interactions determined previously ${ }^{24}$ or in the accompanying article ${ }^{69}$ are shown in blue dashed arrows. B. Schematic representation of a TssM $\mathrm{Cyto}_{\text {dimer }}\left(\mathrm{TssM}_{\mathrm{Cyto} / \mathrm{NTP}}\right.$ are shown in blue, 
$\mathrm{TssM}_{\mathrm{Cyto} / \mathrm{Ct}}$ are shown in green). The cartoon highlights the $\mathrm{TssM}_{\mathrm{Cyto} / \mathrm{Ct}}-\mathrm{TssM}_{\mathrm{Cyto} / \mathrm{Ct}}$ interface and the interaction with TssK, TssG and the cytoplasmic domain of TssL.

\section{LEGEND TO SUPPLEMENTARY FIGURES}

Supplementary Figure S1. Sequence alignment of the cytoplasmic domain from TssM homologues. T-COFFEE sequence alignment of the cytoplasmic domain of TssM of (from top to bottom) entero-aggregative Escherichia coli Sci-1 (EAEC 17-2), Yersinia pseudotuberculosis (IP31758) T6SS-5, Klebsiella pneumoniae, Serratia sp. (strain M24T3), Citrobacter rodentium (sp. A1), Proteus penneri, Myxococcus xanthus (DK1622), Yersinia pseudotuberculosis (IP31758)T6SS-3, Salmonella enterica Typhimurium (LT2), Agrobacterium tumefaciens (C58), Pseudomonas aeruginosa H1-T6SS (PA01), Aeromonas hydrophila (ATCC7966) and Edwardsiella tarda. Local homologies are indicated by a colour code (bad to good from blue to red). The Walker and GTP motifs usually associated with NTPases are indicated, as well as the motifs required for $\mathrm{TssM}_{\text {Cyto }}$ oligomerization and interaction with TssG. The numbering corresponds to the sequence of the EAEC Sci-1 TssM protein. The figure has been prepared with T-COFFEE. ${ }^{67}$

Supplementary Figure S2. Phylogenetic tree of Tsss $\mathbf{M}_{\text {Cyto }}$ homologs. Phylogenetic analysis of the TssM $_{\text {Cyto }}$ sequences shown in Supplementary Fig. S1. The TssM cytoplasmic loops categorize into two phylogenetic clusters, one with complete NTPase domains (orange) and one with NTPase domains but with degenerated Walker motifs (blue). The figure has been prepared with phylogeny.fr. ${ }^{68}$

Supplementary Figure S3. Sequence homology of TssM $\mathbf{M}_{\text {Cyto }}$ sub-domains. A. Sequence alignment of TssM $\mathrm{Cyto/NTP}_{\mathrm{N}}$ with B. thailandensis EngB. B. Sequence alignment of $\mathrm{TssM}_{\mathrm{Cyto} / \mathrm{Ct}}$ with DPY-30. Identical residues are coloured white and underlined in red while strongly similar residues are coloured red. The secondary structures of the EngB and DPY-30 proteins are indicated below the sequences, as well as the percentage of identity and similarity of these proteins with the corresponding TssM $\mathrm{M}_{\text {Cyto }}$ sub-domains.

Supplementary Figure S4. Homology models of TssM $\mathbf{M}_{\mathrm{Cyto}}$ sub-domains. A. Homology models of the EAEC Sci-1 (blue, left panel) and A. tumefaciens (orange, right panel) TssM $\mathrm{Cyto/NTP}_{\text {domains based }}$ on the X-ray structure of the Burkholderia thailandensis EngB GTP-binding protein (PDB ID: 4DHE). The GTP-binding site of the A. tumefaciens TssM ${ }_{\text {Cyto/NTP }}$ is shown in sticks. The models have been generated with HHPred. ${ }^{39}$ B. Homology model of the EAEC TssM $\mathrm{Cyto}_{\mathrm{Ct}}$ domain (green, left panel) based on the X-ray structure of the C-terminal domain of human DPY-30-like protein (PDB ID: 3G36). The residues involved in $\mathrm{TssM}_{\mathrm{Cyt} / \mathrm{Ct}}$ oligomerization are indicated by arrowheads. The model has been generated with Swiss-Model. ${ }^{65}$ The structures of the DPY-30 C-terminal monomer and dimer 
are shown in the middle and right panels respectively. The residues at the DPY-30 dimer interface are indicated by arrowheads. All images have been prepared with Chimera. ${ }^{66}$

Supplementary Figure S5. Topologies of TssM protein homologues. Trans-membrane helix predictions of selected TssM proteins from the indicated strains. Trans-membrane helices are represented by black rectangles whereas $\mathrm{TssM}_{\mathrm{Cyt} / \mathrm{NTP}}$ and $\mathrm{TssM}_{\mathrm{Cyto} / \mathrm{Ct}}$ domains are indicated by blue and green lines respectively. 
A

HMMTop, TMHMM,

TMpred, PHDhtm

Natural Cys

Engineered Cys

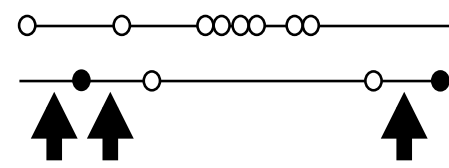

B
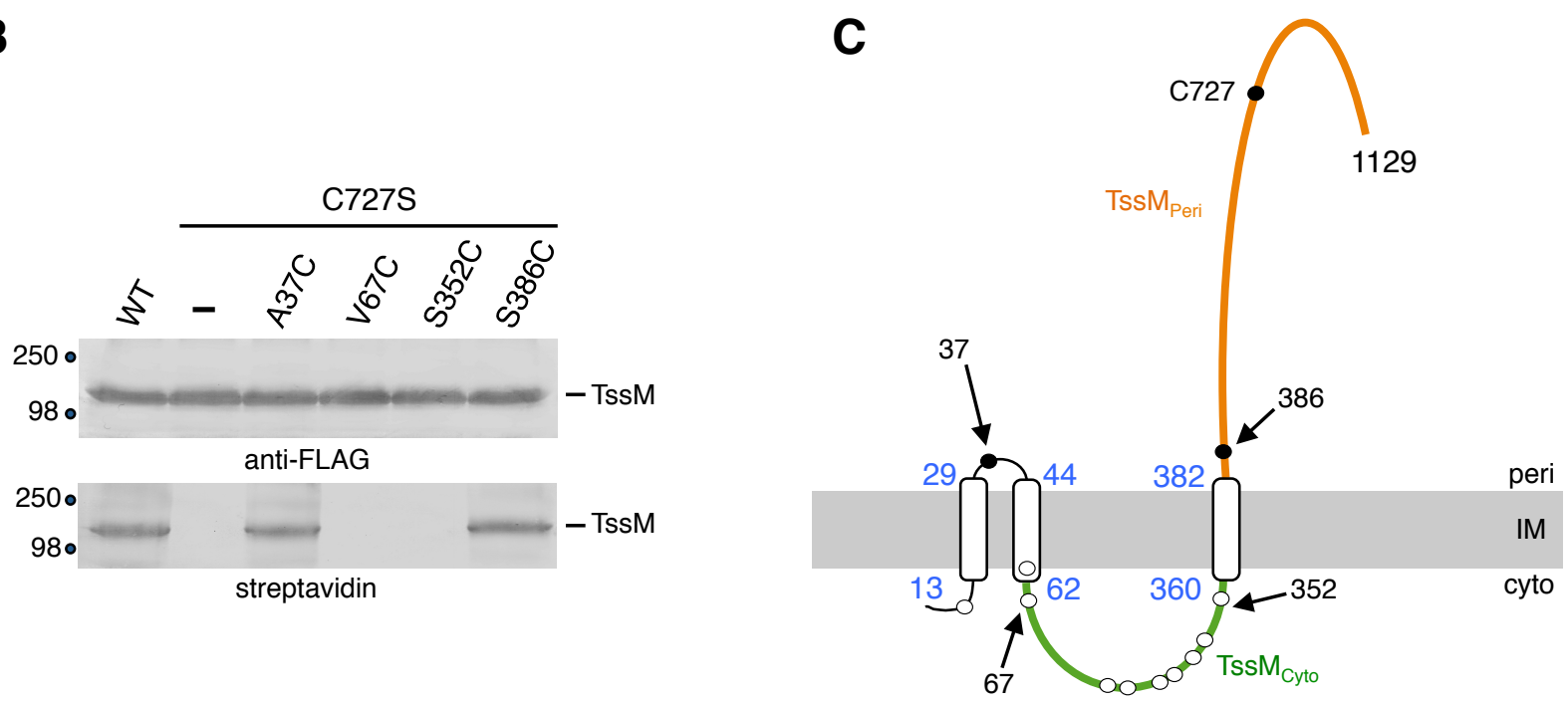
T18-TssM $M_{\text {Cyto }}$

TssM $_{\text {Cyto }}-\mathrm{T} 18$

T18-Pal

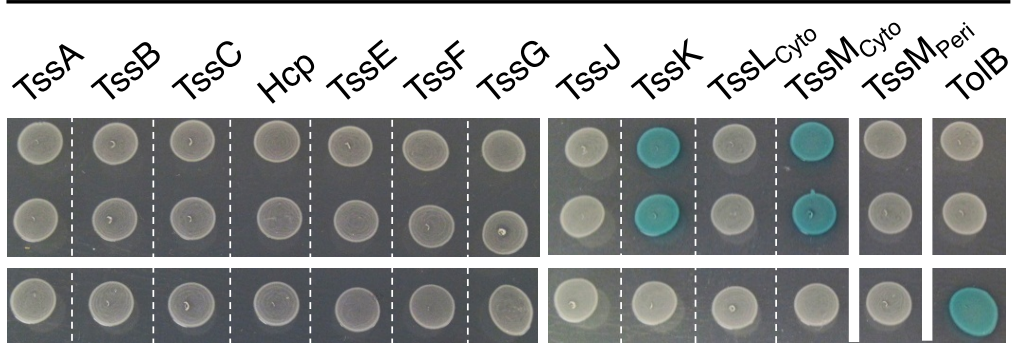

X-T25

T18-TssM $\mathrm{Cyto}_{\text {Cy }}$

TssM $_{\text {Cyto }}-$ T18

T18-Pal

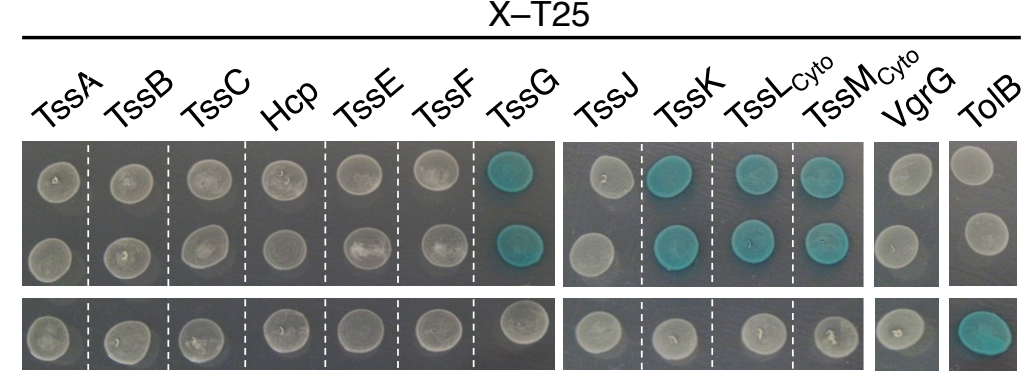




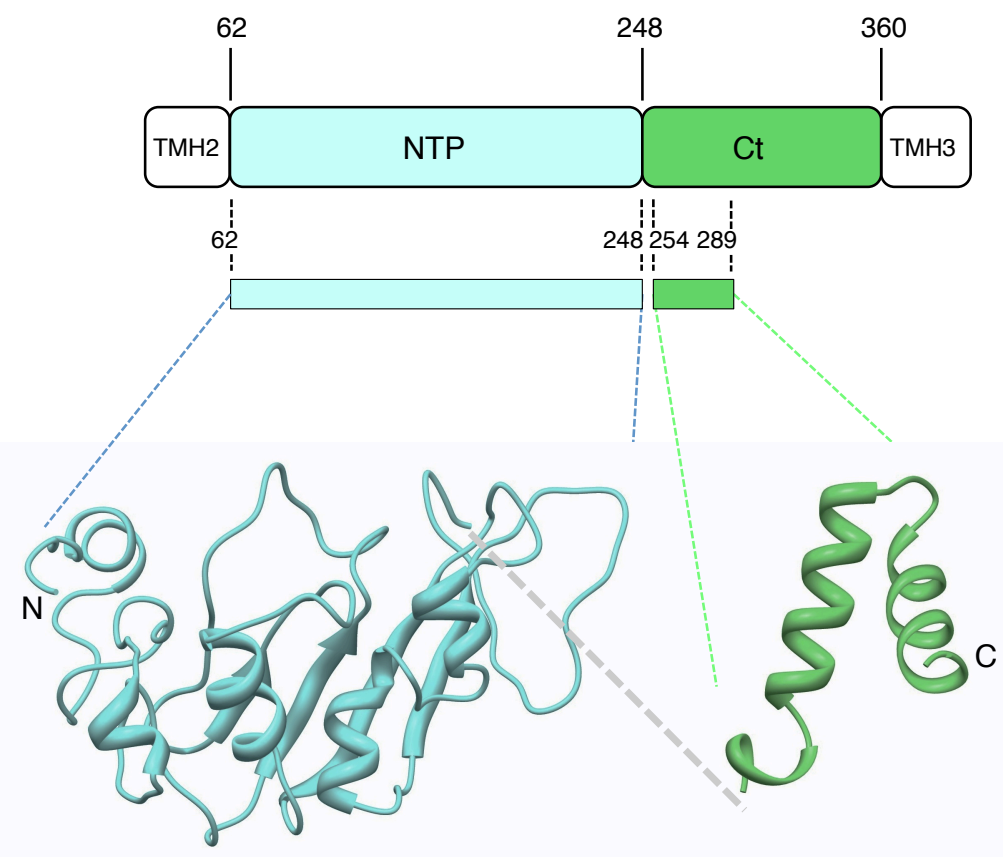

Logger et al. Figure 3 
A

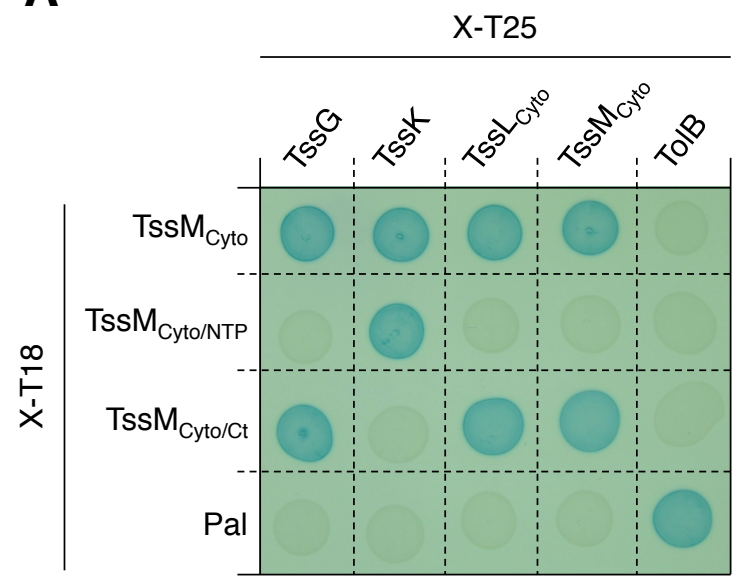

B

$$
\frac{M_{\text {Cyto_FL }}}{\text { Tot IP }} \frac{M_{\text {Cyto/NTP_FL }}}{\text { Tot IP }} \frac{M_{\text {Cyto/CT_FL }}}{\text { Tot IP }}
$$

$50 \bullet$

36

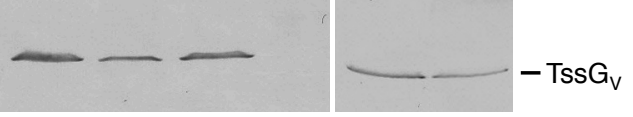

TssK

$$
\frac{M_{\text {Cyto_FL }}}{\text { Tot IP }} \frac{M_{\text {Cyto/NTP_FL }}}{\text { Tot IP }} \frac{M_{\text {Cyto/CT_FL }}}{\text { Tot IP }}
$$

50

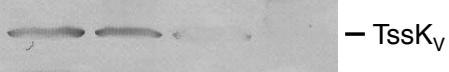


A

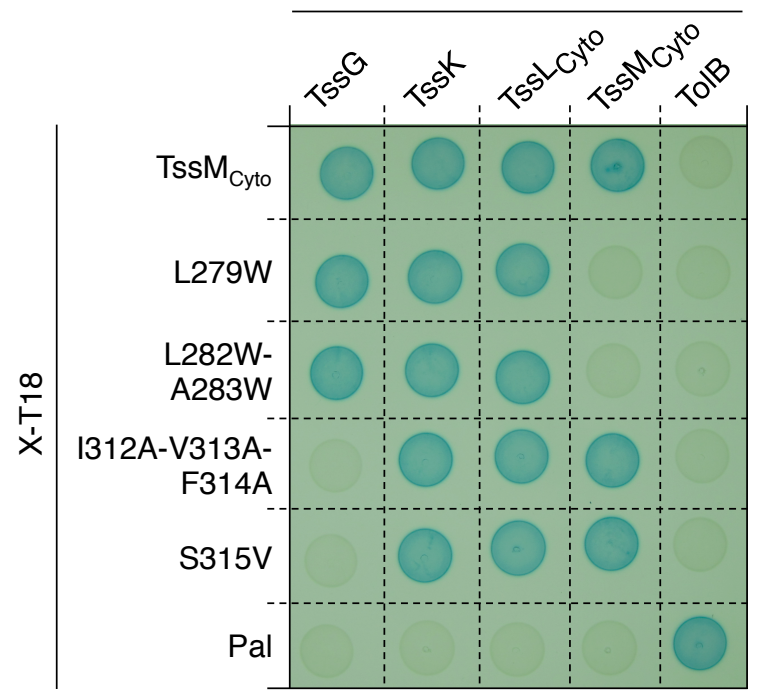

B

TssG

I312A-

L282W- V313A-

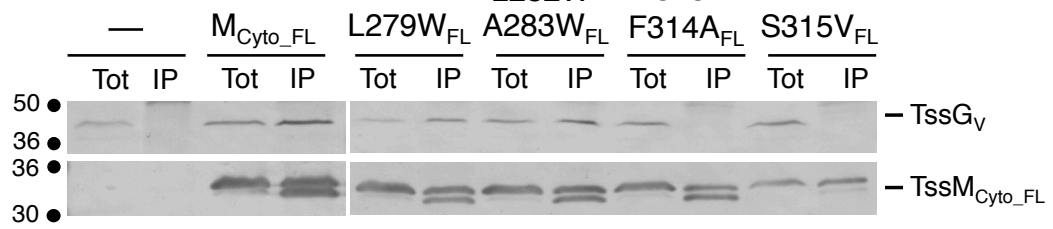

TssM $_{\text {Cyto }}$

I312A-

L282W- V313A-

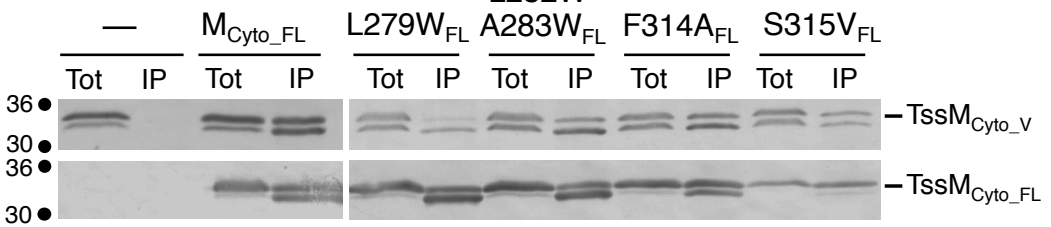



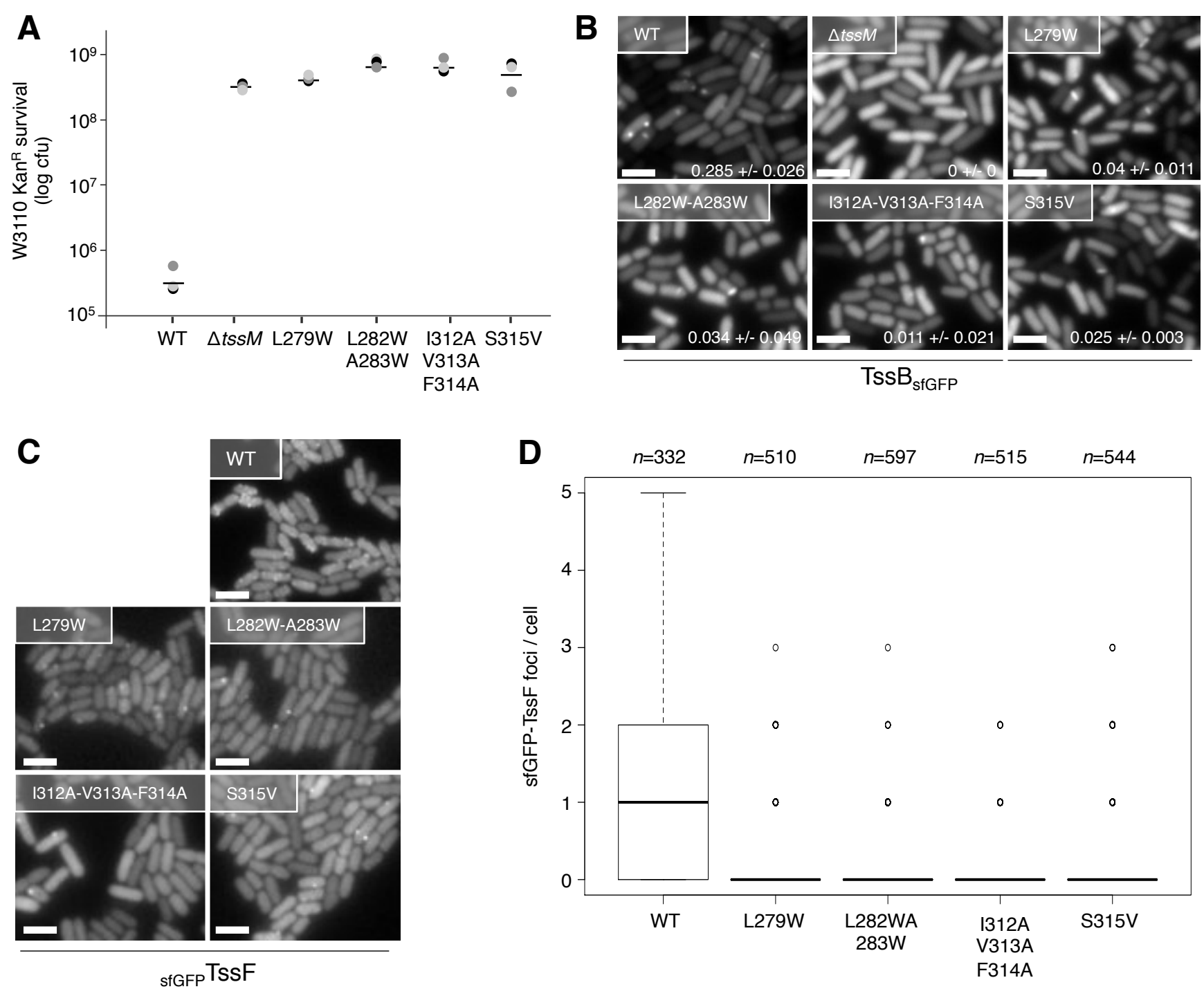


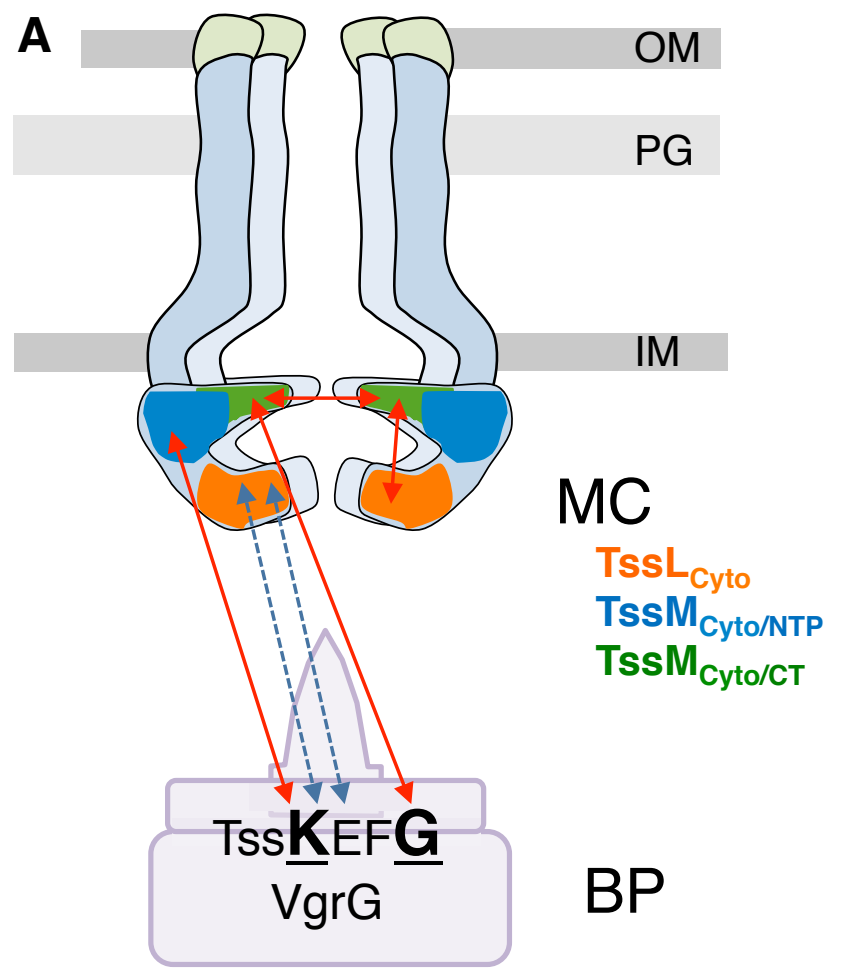

B

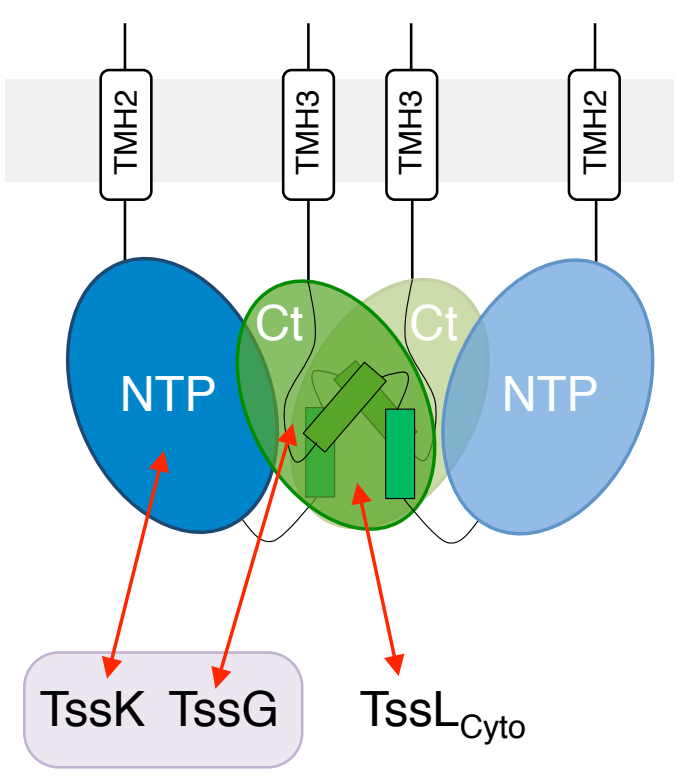




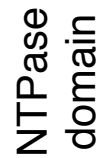
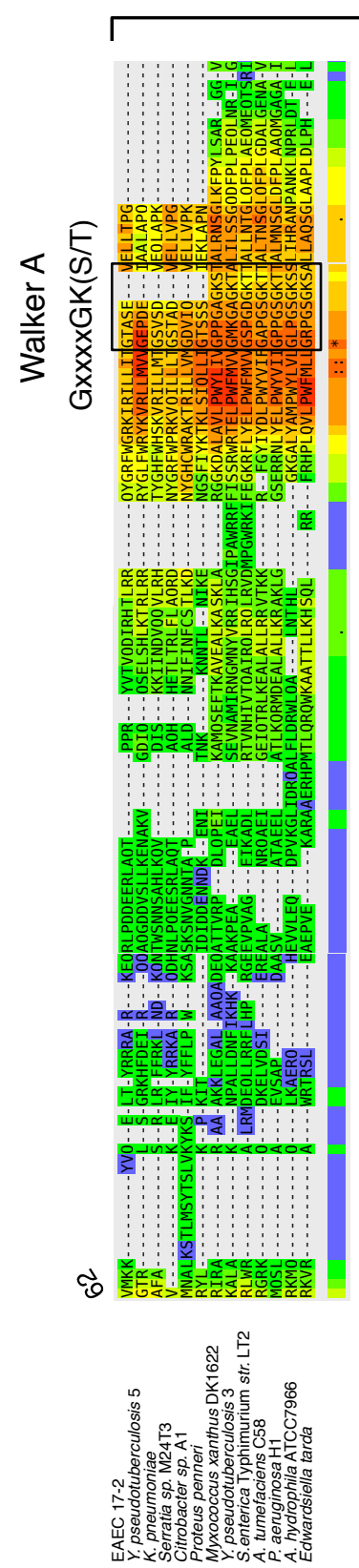

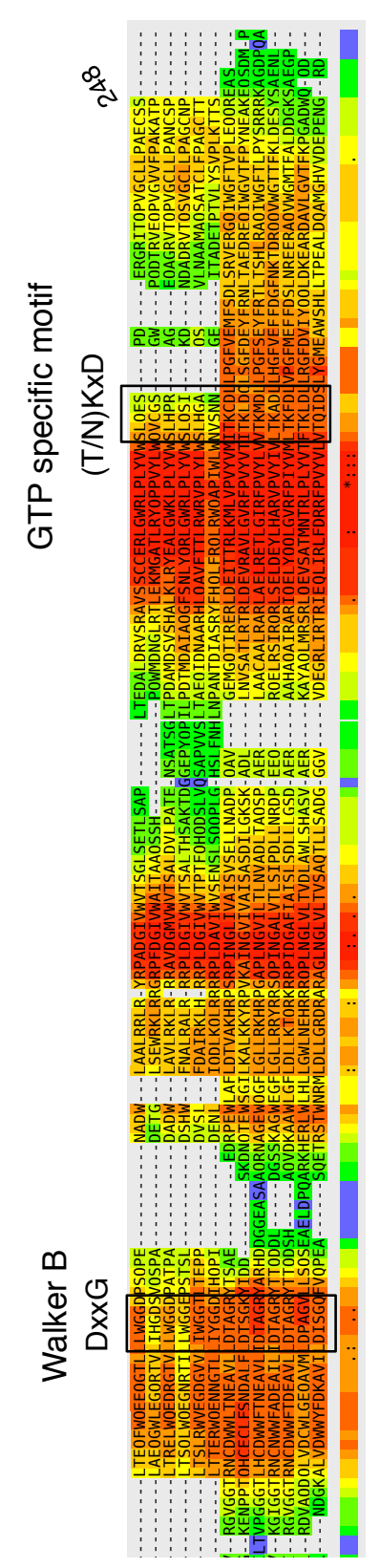

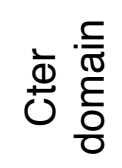
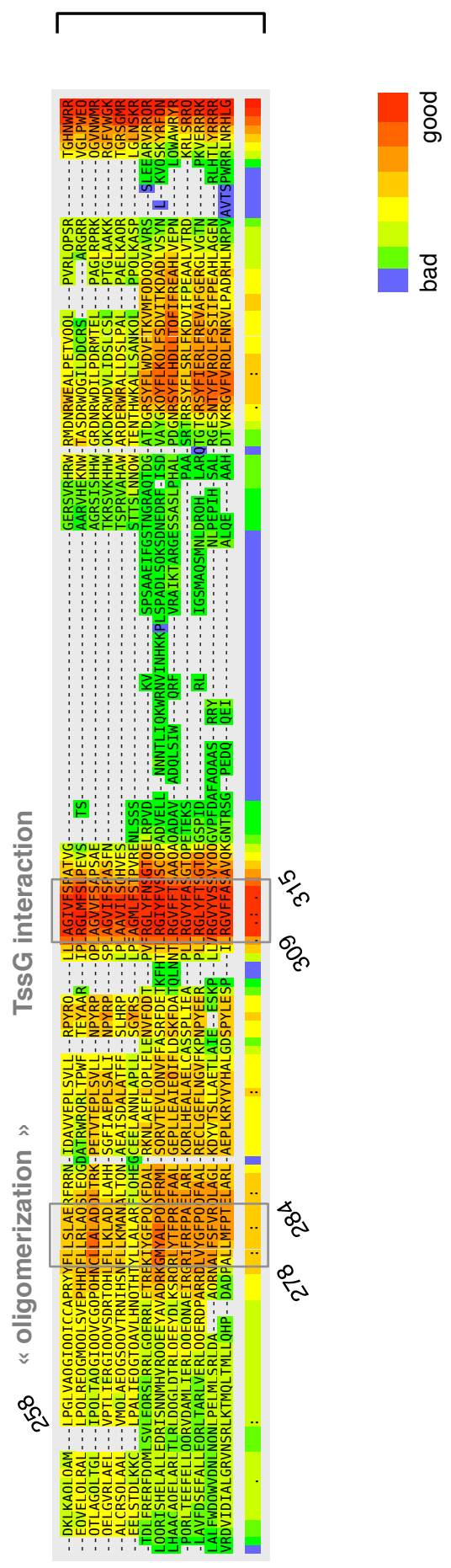


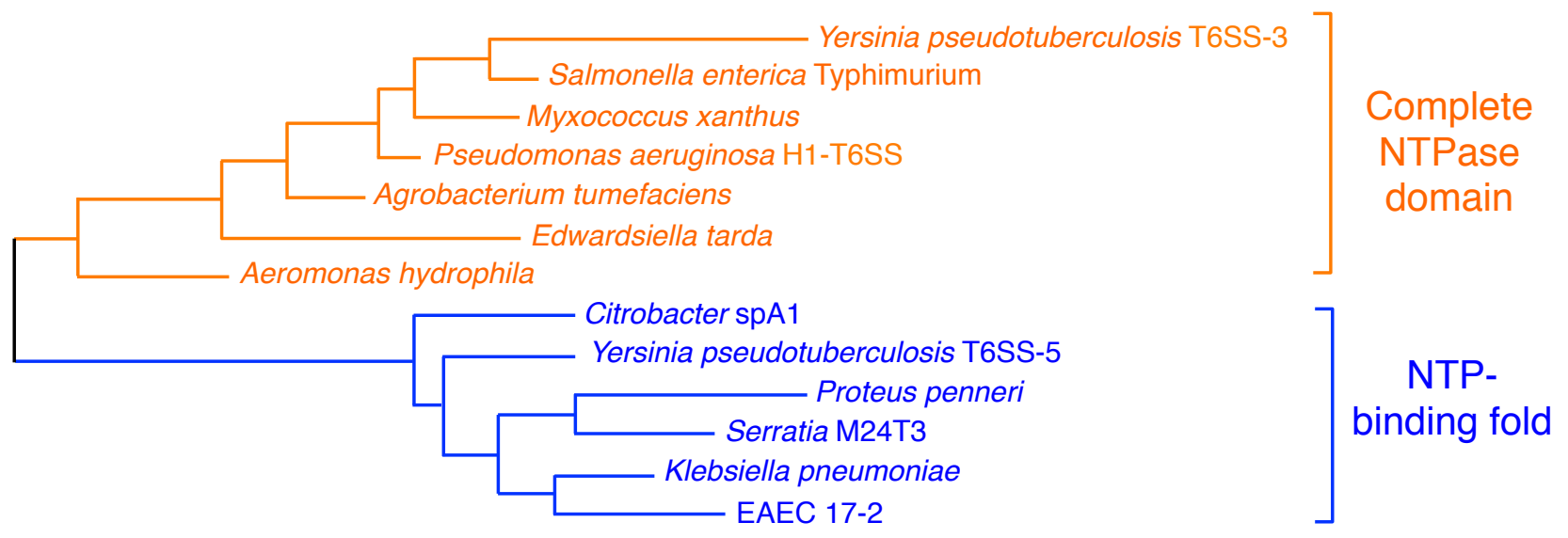


A

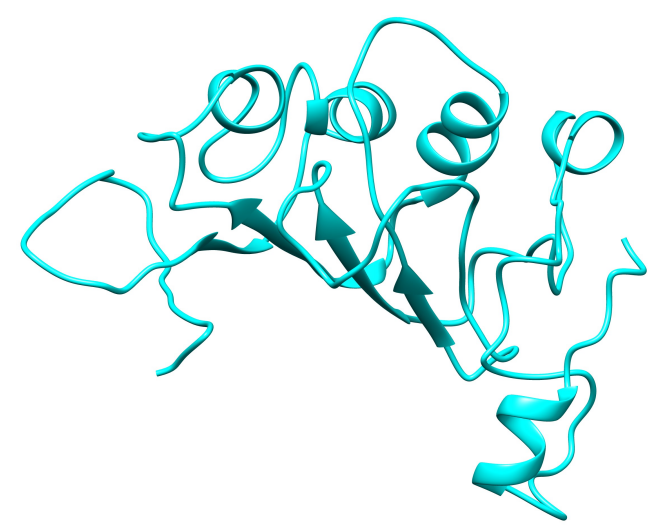

EAEC TssM Cyto/NTP $_{\text {P }}$

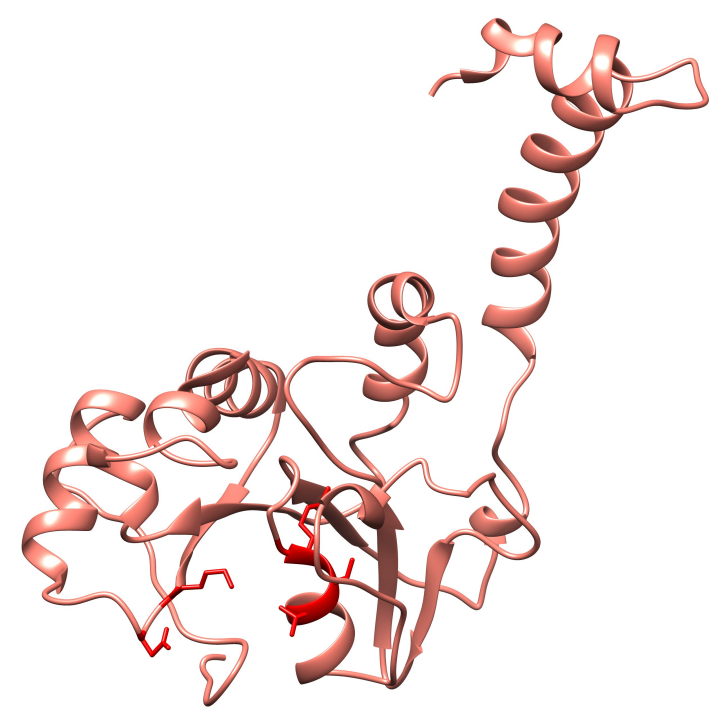

A. tumefaciens TssM Cyto/NTP $_{\text {}}$

B

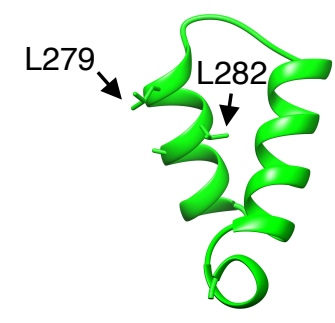

EAEC TssM $\mathrm{Cyto/Ct}_{\mathrm{t}}$

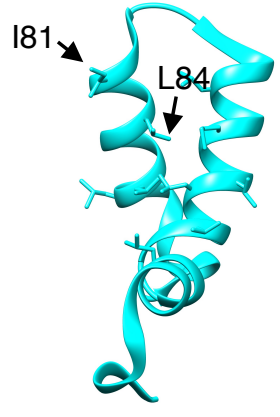

monomer

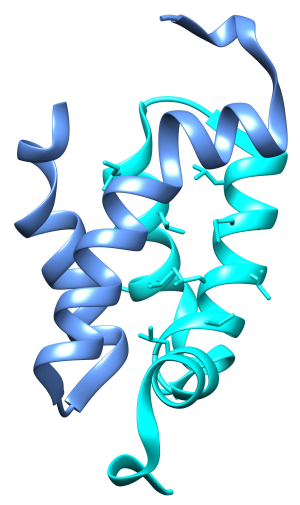

dimer

DPY-30 
EAEC 17-2

A. tumefaciens C58

A. hydrophila

ATCC7966

V. cholerae $\mathrm{O} 395$

$P$. aeruginosa $\mathrm{H} 1$

P. aeruginosa $\mathrm{H} 2$

$P$. aeruginosa $\mathrm{H} 3$

S. marcescens Db11

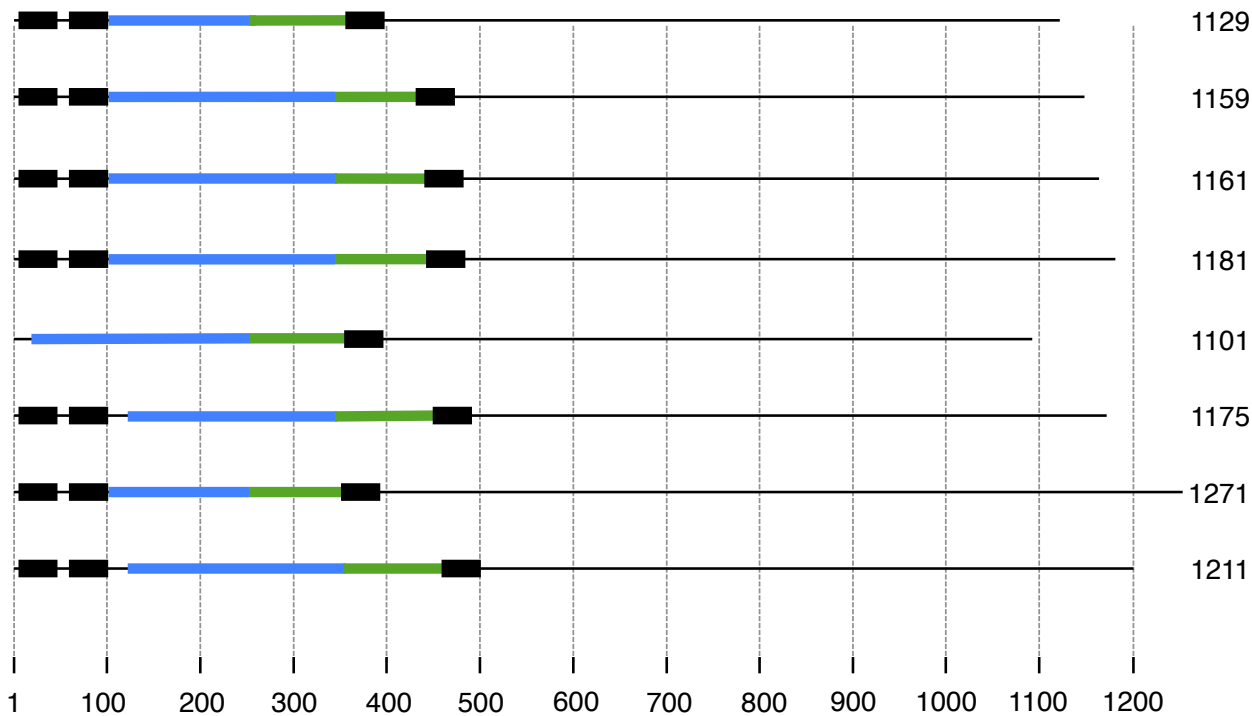




\section{SUPPLEMENTAL DATA}

Molecular dissection of the interface between the Type VI secretion TssM cytoplasmic domain and the TssG baseplate component.

L. Logger, M.S. Aschtgen, M. Guérin, E. Cascales \& E. Durand 


\section{Supplemental Table S1. Strains, plasmids and oligonucleotides used in this study.}

\section{Strains}

Strains

Description and genotype

Source

\section{E. coli K-12}

$\mathrm{DH} 5 \alpha$

W3110

BTH101
F-, $\Delta(\arg F-l a c) \mathrm{U} 169$, phoA, supE44, $\Delta(\mathrm{lacZ}) \mathrm{M} 15$, relA, endA, thi, hsdR

F-, lambda- IN $(r r n D-r r n E) 1 r p h-1$

F-, cya-99, araD139, galE15, galK16, rpsL1 (Str r), hsdR2, mcrA1, mcrB1.
New England Biolabs

Laboratory collection

Karimova et al., 1998

\section{Enteroaggregative E. coli}

\begin{tabular}{|c|c|c|}
\hline $17-2$ & WT enteroaggregative Escherichia coli & A. Darfeuille-Michaud \\
\hline $17-2 \Delta t s s M$ & 17-2 deleted of the $t s s M$ gene of the scil T6SS gene cluster & Aschtgen et al., 2010 \\
\hline $17-2 \operatorname{tss} B-g f p$ & $g f p$ inserted upstream the stop codon of $t s s B$ in $17-2$ & This study \\
\hline $17-2 \Delta t s s M$ tssB-gfp $\Omega$ Kan & $g f p$ inserted upstream the stop codon of $t s s B$ in $17-2 \Delta t s s M$ & This study \\
\hline 17-2 tssB-gfp tssM-L279W & Chromosomal point mutation Leu279-to-Trp substitution of tss $M$ in $17-2$ tssB-gfp & This study \\
\hline 17-2 tssB-gfp tss $M-\mathrm{L} 282 \mathrm{~W}-\mathrm{A} 283 \mathrm{~W}$ & Chromosomal point mutation Leu282 and Ala283-to-Trp substitutions of $t s s M$ in 17-2 tssB-gfp & This study \\
\hline \multicolumn{3}{|c|}{ 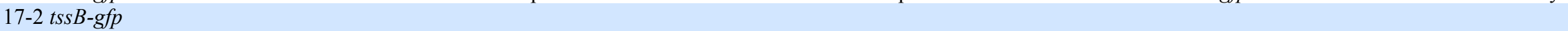 } \\
\hline tss $M-\mathrm{I} 312 \mathrm{~A}-\mathrm{V} 313 \mathrm{~A}-\mathrm{F} 314 \mathrm{~A}$ & Chromosomal point mutation Ile312 and Val313 and Phe314-to-Ala substitutions of tssM in 17-2 tssB-gfp & This study \\
\hline $17-2$ tss $B-g f p \quad t s s M-\mathrm{S} 315 \mathrm{~W}$ & Chromosomal point mutation Ser315-to-Val substitution of tssM in 17-2 tssB-gfp & This study \\
\hline $17-2 g f p-t s s F$ & $g f p$ inserted downstream the start codon of $t s s F$ in $17-2$ & Brunet, Zoued et al., 2015 \\
\hline $17-2 \Delta t s s M g f p$-tssF $\Omega$ Kan & $g f p$ inserted downstream the start codon of $t s s F$ in $17-2 \Delta t s s M$ & This study \\
\hline 17-2 gfp-tssF tssM-L279W & Chromosomal point mutation Leu279-to-Trp substitution of $t s s M$ in $17-2$ gfp-tssF & This study \\
\hline 17- gfp-tssF tss $M$-L282W-A283W & Chromosomal point mutation Leu282 and Ala283-to-Trp substitutions of $t s s M$ in 17-2 gfp-tssF & This study \\
\hline \multicolumn{3}{|c|}{ C } \\
\hline tss $M-\mathrm{I} 312 \mathrm{~A}-\mathrm{V} 313 \mathrm{~A}-\mathrm{F} 314 \mathrm{~A}$ & Chromosomal point mutation Ile 312 and Val313 and Phe314-to-Ala substitutions of $t s s M$ in 17-2 gfp-tssF & This study \\
\hline 17-2 gfp-tssF tssM-S315W & Chromosomal point mutation Ser315 to Val substitution of $t s s M$ in 17-2 $g f p$-tssF & This study \\
\hline
\end{tabular}




\section{Plasmids}

\section{Vectors for chromosomal insertions}

pKD4 One-step gene inactivation vector, $\mathrm{Kan}^{\mathrm{R}}$

$\mathrm{pg} f \mathrm{p}-\mathrm{KD} 4$

pKOBEG superfolder- $g f p$ cloned upstream the 3' -FRT site of pKD4. Used for chromosomal C-terminal GFP fusion

Recombination vector, phage $\lambda$ rec $\beta \alpha$ operon under the control of the $\mathrm{pBAD}$ promoter, $\mathrm{Cm}^{\mathrm{R}}$
Datsenko \& Wanner, 2000

Brunet, Zoued et al., 2015

Chaveroche et al., 2000

\section{Expression vectors}

pUA66-rrnb

pASK-IBA37(+)

pASK-IBA37-FLAGTssM

pIBA37-fLAGTssM-C727S

pIBA37-FLAG TssM-C727S-A37C

pIBA37-FLAG TssM-C727S-V67C

pIBA37-FLAGTssM-C727S-S352C

pIBA37-FLAG ${ }^{\text {TsSM-C72 }}$

pIBA37-TssM Cyto $-\mathrm{NTP}_{\text {FLAG }}$

pIBA37-TssM Cyto $_{\text {-Cter }}$ FLAG

pIBA37-TssM Cyto $_{\text {-L279W }}$ FLAG

pIBA37-TssM Cyto $-\mathrm{L} 282 \mathrm{~W}$

$-\mathrm{A} 283 \mathrm{~W}_{\mathrm{FLAG}}$

pIBA37-TssM ${ }_{\text {Cyto }}-\mathrm{I} 312 \mathrm{~A}$

-V313A-F314A

pIBA37-TssM $\mathrm{Cyto}_{\text {Cyt }}-\mathrm{S} 315 \mathrm{~V}_{\mathrm{FLAG}}$ pBAD33

pBAD33-TssM Cyto_vsv-G $_{\text {- }}$

pBAD33-TssG $\mathrm{VSV}_{\mathrm{V}-\mathrm{G}}$

pBAD33-TssK $K_{V S V-G}$
$P_{r r n B}: \because g f p m u t 2$ transcriptional fusion in pUA66 cloning vector, Ptet, $\mathrm{fl}$ origin, Amp

$s c i-1$ tss $M$ carrying N-terminal FLAG tag cloned into pASK-IBA37

Introduction of the TssM Cys727-to-Ser substitution into pIBA37-FLAGTssM

ntroduction of the TssM Ala37-to-Cys substitution into pIBA37-f 3 TssM-C727S

Introduction of the TssM Val67-to-Cys substitution into pIBA37-FLAGTssM-C727S

ntroduction of the TssM Ser352-to-Cys substitution into pIBA37-FLAGTssM-C727S

Introduction of the TssM Ser386-to-Cys substitution into pIBA37-FLAG TssM-C727 $s c i-1$ tss $M$ residues 62-360 cloned into pASK-IBA37, C-terminal FLAG epitope sci-1 tss $M$ residues 62-273 cloned into pASK-IBA37, C-terminal FLAG epitope sci-1 tss $M$ residues 274-360 cloned into pASK-IBA37, C-terminal FLAG epitope Introduction of the TssM Leu279-to-Trp substitution into pIBA37-TssM Cyto FLAG $_{\text {f }}$

Introduction of the TssM Leu282 and Ala283-to-Trp substitutions into pIBA37-TssM

Zaslaver et al,, 2006

IBA Technology

Aschtgen et al., 2010

This study

This study

This study

This study

This study

Zoued et al., 2013

This study

This study

This study

Introduction of the TssM Ile312 and Val313 and Phe314-to-Ala substitutions into pIBA37-TssM Cyto FLAG $_{\text {. }}$

This study Introduction of the TssM Ser 315-to-Val substitution into pIBA37-TssM $\mathrm{Cyto}_{\text {_FLAG }}$ cloning vector, $\mathrm{P} 15 \mathrm{~A}$ origin, Para, araC, $\mathrm{Cm}^{\mathrm{R}}$

This study This study scil tss $M_{C y t o}$ cloned into pBAD33, C-terminal VSV-G epitope scil tss $G$ cloned into pBAD33, C-terminal VSV-G epitope Guzman et al., 1995 This study scil tssK cloned into pBAD33, C-terminal VSV-G epitope
Brunet, Zoued et al., 2015 Brunet, Zoued et al., 2015 


\begin{tabular}{|c|c|c|}
\hline pT18-FLAG & Bacterial two-hybrid vector, ColE1 origin, Plac, T18 fragment of Bordetella pertussis CyaA, Amp ${ }^{\mathrm{R}}$ & Battesti \& Bouveret, 2008 \\
\hline pTssM $_{\text {Cyto }}-\mathrm{T} 18$ & Cytoplasmic region of $t s s M$ (TssM ${ }_{62-360}$ fragment) cloned upstream T18 in pT18-FLAG & Zoued et al., 2013 \\
\hline pT18-TssM Cyto $_{1}$ & Cytoplasmic region of $t s s M$ ( $\operatorname{TssM}_{62-360}$ fragment) cloned downstream T18 in pT18-FLAG & Zoued et al., 2013 \\
\hline pTssM $\mathrm{Cyto}_{\text {-NTP-T18 }}$ & sci- 1 tss $M$ residues $62-273$ cloned upstream T18 into pT18-FLAG & This study \\
\hline pTssM $\mathrm{Cyto}_{\text {-Cter-T18 }}$ & sci-1 tss $M$ residues $274-360$ cloned upstream T18 into pT18-FLAG & This study \\
\hline pTssM $_{\text {Cyto }}-\mathrm{L} 279 \mathrm{~W}-\mathrm{T} 18$ & 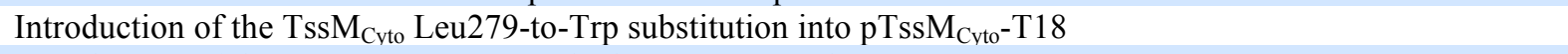 & This study \\
\hline $\begin{array}{l}\text { pTssM } \\
\text { Cyto-L282W-A283W-T18 } \\
\text { This study }\end{array}$ & Introduction of the TssM $\mathrm{Cyto}_{\text {Leu282 and Ala283-to-Trp substitutions into pTssM }} \mathrm{Cyto}-\mathrm{T} 18$ & \\
\hline $\begin{array}{l}\text { pTssM } \\
\text { Cyto-I312A-V313A- } \\
\text { F314A-T18 }\end{array}$ & Introduction of the TssM ${ }_{\text {Cyto }}$ Ile312 and Val313 and Phe314-to-Ala substitutions into pTssM $\mathrm{Cyto}_{\text {-T18 }}$ & This study \\
\hline pTssM $_{\text {Cyto }}-\mathrm{S} 315 \mathrm{~V}-\mathrm{T} 18$ & Introduction of the TssM ${ }_{\text {Cyto }}$ Ser315-to-Val substitution into pTssM $\mathrm{Cyto}_{\mathrm{O}} \mathrm{T} 18$ & This study \\
\hline pT18-Pal & pal cloned downstream the T18 coding sequence in pT18-FLAG & Battesti \& Bouveret, 2008 \\
\hline pT25-FLAG & Bacterial two-hybrid vector, P15A origin, Plac, T25 fragment of Bordetella pertussis CyaA, Kan ${ }^{\mathrm{R}}$ & Battesti \& Bouveret, 2008 \\
\hline pT25-TssA & tss $A$ cloned downstream the T25 coding sequence in pT25-FLAG & Zoued et al., 2013 \\
\hline pTssA-T25 & tss $A$ cloned upstream the T25 coding sequence in pT25-FLAG & Zoued et al., 2013 \\
\hline pT25-TssB & tss $B$ cloned downstream the T25 coding sequence in pT25-FLAG & Zoued et al., 2013 \\
\hline pTssB-T25 & tss $B$ cloned upstream the T25 coding sequence in pT25-FLAG & Zoued et al., 2013 \\
\hline pT25-TssC & tss $C$ cloned downstream the T25 coding sequence in pT25-FLAG & Zoued et al., 2013 \\
\hline pTssC-T25 & tss $C$ cloned upstream the T25 coding sequence in pT25-FLAG & Zoued et al., 2013 \\
\hline pT25-Hcp & $h c p$ cloned downstream the T25 coding sequence in pT25-FLAG & Zoued et al., 2013 \\
\hline pHcp-T25 & $h c p$ cloned upstream the T25 coding sequence in pT25-FLAG & Zoued et al., 2013 \\
\hline pT25-TssE & tssE cloned downstream the T25 coding sequence in pT25-FLAG & Zoued et al., 2013 \\
\hline pTssE-T25 & tssE cloned upstream the T25 coding sequence in pT25-FLAG & Zoued et al., 2013 \\
\hline pT25-TssF & tss $F$ cloned downstream the T25 coding sequence in pT25-FLAG & Zoued et al., 2013 \\
\hline pTssF-T25 & $t s s F$ cloned upstream the T25 coding sequence in pT25-FLAG & Zoued et al., 2013 \\
\hline pT25-TssG & tss $G$ cloned downstream the T25 coding sequence in pT25-FLAG & Zoued et al., 2013 \\
\hline pTssG-T25 & tss $G$ cloned upstream the T25 coding sequence in pT25-FLAG & Zoued et al., 2013 \\
\hline pT25-VgrG & $\operatorname{vgr} G$ cloned downstream the T25 coding sequence in pT25-FLAG & Zoued et al., 2013 \\
\hline pVgrG-T25 & $\operatorname{vgr} G$ cloned upstream the T25 coding sequence in pT25-FLAG & Zoued et al., 2013 \\
\hline pT25-TssJ $\mathrm{sol}_{\mathrm{N}}$ & $t s s J_{s o l}\left(\mathrm{Tss}_{2-152}\right.$ fragment of the processed form) cloned downstream the T25 coding sequence in pT25-FLAG & Zoued et al., 2013 \\
\hline $\mathrm{pTss}_{\mathrm{sol}}-\mathrm{T} 25$ & $t s s J_{s o l}\left(\mathrm{Tss}_{2-152}\right.$ fragment of the processed form) cloned upstream the T25 coding sequence in pT25-FLAG & Zoued et al., 2013 \\
\hline pT25-TssK & tss $K$ cloned downstream the T25 coding sequence in pT25-FLAG & Zoued et al., 2013 \\
\hline pTssK-T25 & tss $K$ cloned upstream the T25 coding sequence in pT25-FLAG & Zoued et al., 2013 \\
\hline pT25-TssL $L_{\text {Cyto }}$ & Cytoplasmic fragment of $t s s L$ (TssL $\mathrm{T}_{1-184}$ fragment) cloned downstream the T25 coding sequence in pT25-FLAG & Durand et al., 2012 \\
\hline $\mathrm{pTssL}_{\mathrm{Cyto}}-\mathrm{T} 25$ & Cytoplasmic fragment of tssL (TssL $\mathrm{T}_{1-184}$ fragment) cloned upstream the $\mathrm{T} 25$ coding sequence in pT25-FLAG & Durand et al., 2012 \\
\hline pT25-TssM Cyto $_{1}$ & Cytoplasmic fragment of $t s s M$ (TssM ${ }_{62-360}$ fragment) cloned downstream T25 in pT25-FLAG & Zoued et al., 2013 \\
\hline pTssM $_{\text {Cyto }}-\mathrm{T} 25$ & Cytoplasmic fragment of $t s s M$ (TssM ${ }_{62-360}$ fragment) cloned upstream T25 in pT25-FLAG & Zoued et al., 2013 \\
\hline
\end{tabular}




\section{Chromosomal mutagenesis vectors}

pKO3

pKO3-tss $M$

pKO3-tssM- L279W

pKO3-tss $M$-L282W-A283W

pKO3-tss $M-\mathrm{I} 312 \mathrm{~A}-\mathrm{V} 313 \mathrm{~A}-\mathrm{F} 314 \mathrm{~A}$

pKO3-tss $M$-S315W $\operatorname{sac} B, \operatorname{rep} A\left(\mathrm{pSC} 101^{t s}\right), \mathrm{M} 13$ origin, $\mathrm{Cm}^{\mathrm{R}}$

Region of $t s s M$ cloned into the $\mathrm{pKO} 3$ vector

Link et al., 1997 This study

This study

This study

This study

This study
Introduction of the TssM Leu282 and Ala283-to-Trp substitutions into pKO3-tssM

Introduction of the TssM Ser315-to-Val substitution into pKO3-tssM 


\section{Oligonucleotides}

Name

Destination

Sequence $\left(5^{\prime} \rightarrow 3^{\prime}\right)$

For site-directed mutagenesis $^{\text {a }}$

\begin{tabular}{|c|c|c|}
\hline M-C727S-5 & pASK-IBA37-FLAGTssM & GAATACGCTGGCGGTTCAGGGATCCACTGGCCAGCCCCGGGAAG \\
\hline M-C727S-3 & pASK-IBA37-FLAGTssM & CTTCCCGGGGCTGGCCAGTGGATCCCTGAACCGCCAGCGTATTC \\
\hline M-A36C-5 & pIBA37-FLAGTssM-C727S & GATAACCCGATATGGAGCATACCTAGGGTGTGAAACACGCCGGGATCAAATAC \\
\hline M-A36C-3 & pIBA37-FLAGTssM-C727S & GTATTTGATCCCGGCGTGTTTCACACCCTAGGTATGCTCCATATCGGGTTATC \\
\hline M-V67C-5 & pIBA37-FLAGTssM-C727S & CTGCCTGTGATGAAAAAATATTGTCAGGAACTGACATATCGAC \\
\hline M-V67C-3 & pIBA37-FLAG TssM-C727S & GTCGATATGTCAGTTCCTGACAATATTTTTTCATCACAGGVAG \\
\hline M-S352C-5 & pIBA37-FLAGTssM-C727S & AATTACCTGTCCGGCTACAGCCCTGCAGGACAGGTCATAACTGGCGCAG \\
\hline M-S352C-3 & pIBA37-FLAG TssM-C727S & CTGCGCCAGTTATGACCTGTCCCTGCAGGGCTGTAGCCGGACAGGTAATTG \\
\hline M-S386C-5 & pIBA37-FLAGTssM-C727S & GGTGGTTTCCTTTCTGGCAAATCGATGTCTGGTTGCTGAAGTCAG \\
\hline M-S386C-3 & pIBA37-FLAGTssM-C727S & CTGTACTTCAGCAACCAGACATCGATTTGCCAGAAAGGAAACCACC \\
\hline A-L279W & $\mathrm{pTssM}_{\mathrm{Cyto}}-\mathrm{T} 18, \mathrm{pIBA} 37-\mathrm{TssM}_{\mathrm{Cyto} \_\mathrm{FL}}$ or pKO3-tss $M$ & CCCTCGGTATTATTTTTGGTTGTCGCTGGCAGAGC \\
\hline B-L279W & pTssM $_{\text {Cyto }}-\mathrm{T} 18, \mathrm{pIBA} 37-\mathrm{TssM}_{\mathrm{Cyto}} \mathrm{FL}$ or $\mathrm{pKO} 3-t s s M$ & ATAATACCGAGGGGCACAGCATATCTGCTG \\
\hline A-L282W-A283W & $\mathrm{pTssM}_{\mathrm{Cyto}}-\mathrm{T} 18, \mathrm{pIBA} 37-\mathrm{TssM}_{\mathrm{Cyto} \_\mathrm{FL}}$ or pKO3-tssM & CCCTCGGTATTATTTTTTGTTGTCGTGGTGGGAGCGATTCAGACGAA \\
\hline B- L282W-A283W & pTssM $_{\text {Cyto }}-\mathrm{T} 18, \mathrm{pIBA} 37-\mathrm{TssM}_{\mathrm{Cyto}} \mathrm{FL}$ or $\mathrm{pKO} 3-t s s M$ & ATAATACCGAGGGGCACAGCATATCTGCTG \\
\hline A-I312A-V313A-F314A & $\mathrm{pTssM}_{\mathrm{Cyto}}-\mathrm{T} 18, \mathrm{pIBA} 37-\mathrm{TssM}_{\mathrm{Cyto} \_\mathrm{FL}}$ or pKO3-tssM & GTTGTTGCTGGCGGGTGCGGCGGCGAGTCCGGCAACTGTCG \\
\hline B- I312A-V313A-F314A & $\mathrm{pTssM}_{\mathrm{Cyto}}-\mathrm{T} 18, \mathrm{pIBA} 37-\mathrm{TssM}_{\mathrm{Cyto}}$ FL or pKO3-tssM & CGCCAGCAACAACTGACGGTATGGACGGAG \\
\hline A- S315V & $\mathrm{pTssM}_{\mathrm{Cyto}}-\mathrm{T} 18, \mathrm{pIBA} 37-\mathrm{TssM}_{\mathrm{Cyto} \_\mathrm{FL}}$ or pKO3-tss $M$ & GTTGTTGCTGGCGGGTATTGTTTTCGTGCCGGCAACTGTCGGCG \\
\hline B- S315V & $\mathrm{pTssM}_{\mathrm{Cyto}}-\mathrm{T} 18, \mathrm{pIBA} 37-\mathrm{TssM}_{\mathrm{Cyto} \_\mathrm{FL}}$ or $\mathrm{pKO} 3-t s s M$ & CGCCAGCAACAACTGACGGTATGGACGGAG \\
\hline
\end{tabular}

$\underline{\text { For plasmid construction }}^{\text {b,c, }}$

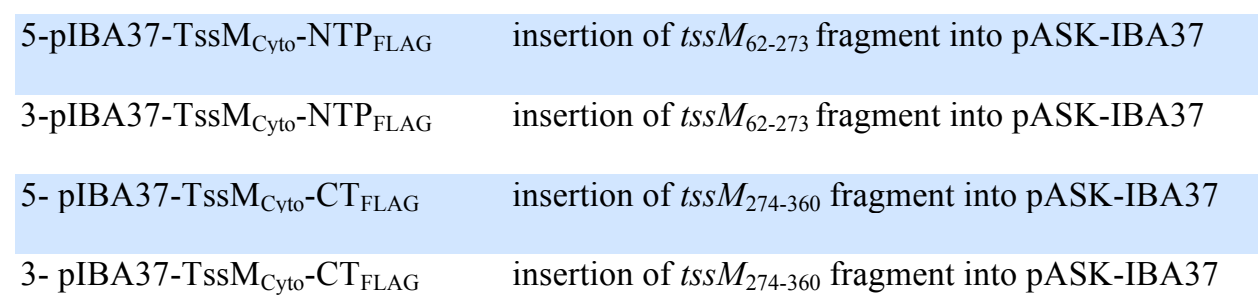

GACAAAAATCTAGAAATAATTTTGTTTAACTTTAAGAAGGAGATATACA AATGGTGATGAAAAAATATGTTCAGGAACTGACATATC

GATGGTGATGGTGATGCGATCCTCTGCTAGCTTATTTATCATCGTCGTCTT TATAATCGGCACAGCATATCTGCTGTATCCC

GACAAAAATCTAGAAATAATTTTGTTTAACTTTAAGAAGGAGATATACA AATGCCTCGGTATTATTTTTTGTTGTCGCTGGC

GATGGTGATGGTGATGCGATCCTCTGCTAGCTTACTTGTCATCGTCGTCCT TATAATCTCTGCGCCAGTTATGACCTGTGCGGGA 


\begin{tabular}{|c|c|c|}
\hline T25T18C-5-TssM Cyto $_{\text {-NTP }}$ & insertion of $t s s M_{62-273}$ fragment downstream the T18 domain & $\frac{\text { CGGATAACAATTTCACACAGGAAACAGCTATGACCATGGTGAT }}{\text { GAAAAAATATGTTCAGGAACTGACATATCG }}$ \\
\hline T18C-3- TssM $\mathrm{Cyto}_{\mathrm{NTP}}$ & insertion of $t s s M_{62-273}$ fragment downstream the T18 domain & $\frac{\text { CCTCGCTGGCGGCTAAGCTTGGCGTAATGGCACAGCATATCTG }}{\text { CTGTATCCC }}$ \\
\hline T25T18C-5-TssM $\mathrm{Cyto}_{\text {-CT }}$ & insertion of $t s s M_{274-360}$ fragment downstream the T18 domain & $\frac{\text { CGGATAACAATTTCACACAGGAAACAGCTATGACCATGCCTCG }}{\text { GTATTATTTTTTGTTGTCGCTGGC }}$ \\
\hline T18C-3- TssM ${ }_{\text {Cyto }}-\mathrm{CT}$ & insertion of $t s s M_{274-360}$ fragment downstream the T18 domain & $\frac{\text { CCTCGCTGGCGGCTAAGCTTGGCGTAATTCTGCGCCAGTTATGA }}{\text { CCTGTGCG }}$ \\
\hline 5-pBAD-TssM $\mathrm{M}_{\text {Cyto }}$ & insertion of $t s s M_{274-360}$ fragment into pBAD33 & $\begin{array}{l}\text { ACTGTTTCTCCATACCCGTTTTTTTGGGCTAGCAGGAGGTATTAC } \\
\text { CCATGGTGATGAAAAAATATGTTCAGGAACTGACATATCGAC }\end{array}$ \\
\hline 3-pBAD-TssM ${ }_{\mathrm{Cyto}}-\mathrm{VSVG}$ & insertion of $t s s M_{62-360}$ fragment into pBAD33 & $\begin{array}{l}\text { ACTCTAGAGGATCCCCGGGTACCTTA TTTTCCTAATCTATTCATTTCA } \\
\text { TATCTGTATATCTGCGCCAGTTATGACCTGTGCGGGA }\end{array}$ \\
\hline 5-pKO3-BamHI-TssM ${ }_{\text {Cyto }}$ & insertion of $t s s M$ fragment into $\mathrm{pKO} 3$ & SATCCCGACGCAGGGCCCGTAAG \\
\hline 3-pKO3-SalI-TssM ${ }_{\text {Cyto }}$ & insertion of $t s s M$ fragment into $\mathrm{pKO} 3$ & CGACCTGTGGCCATTCCCGCATGAG \\
\hline \multicolumn{3}{|l|}{ For strain construction $^{\mathrm{e}}$} \\
\hline 5-tssB-gfp & insertion of $g f p$-mut 2 at the $3^{\prime}$ end of tss $B$ & $\begin{array}{l}\text { ICTGAGTCAGACGCTGCGTGATGAACTGCGTGCACTGGTGCCGGA } \\
\text { AAGGCGGCAGCGGCCGGCGGAGGG }\end{array}$ \\
\hline 3-tssB-gfp & insertion of $g f p-m u t 2$ at the $3^{\prime}$ end of tss $B$ & $\begin{array}{l}\text { צTTCTTTTCTTTCTGTACAGACATCAGCATTTTCTCTCGTAATCCG } \\
\text { TAAACATATGAATATCCTCCTTAGTTCCTATTCCGAAGTTCC }\end{array}$ \\
\hline
\end{tabular}

\footnotetext{
${ }^{a}$ Mutagenesized codon in Bold.

${ }^{\mathrm{b}}$ Sequence annealing on the target plasmid underlined.

${ }^{\mathrm{c}}$ FLAG or VSV-G epitope coding sequence italicized.

${ }^{\mathrm{d}}$ Restriction sites in Bold.

${ }^{\mathrm{e}}$ Sequences corresponding to the downstream and upstream regions of the gene to be inserted underlined
} 